\title{
A unified phase transition picture of the charged topological black hole in Hořava-Lifshitz gravity
}

\author{
Jie-Xiong Mo, ${ }^{a, b}$ Xiao-Xiong Zeng, ${ }^{c, a}$ Gu-Qiang Li, ${ }^{b}$ Xin Jiang, ${ }^{a}$ Wen-Biao Liu ${ }^{a, 1}$ \\ ${ }^{a}$ Department of Physics, Institute of Theoretical Physics, Beijing Normal University, \\ Beijing, 100875, China \\ ${ }^{b}$ Institute of Theoretical Physics, Zhanjiang Normal University, \\ Zhanjiang, Guangdong, 524048, China \\ ${ }^{c}$ School of Science, Chongqing Jiaotong University, \\ Nanan, Chongqing, 400074, China
}

\begin{abstract}
Aiming at a unified phase transition picture of the charged topological black hole in Hořava-Lifshitz gravity, we investigate this issue not only in canonical ensemble with the fixed charge case but also in grand-canonical ensemble with the fixed potential case. We firstly perform the standard analysis of the specific heat, the free energy and the Gibbs potential, and then study its geometrothermodynamics. It is shown that the local phase transition points not only witness the divergence of the specific heat, but also witness the minimum temperature and the maximum free energy or Gibbs potential. They also witness the divergence of the corresponding thermodynamic scalar curvature. No matter which ensemble is chosen, the metric constructed can successfully produce the behavior of the thermodynamic interaction and phase transition structure while other metrics failed to predict the phase transition point of the charged topological black hole in former literature. In grand-canonical ensemble, we have discovered the phase transition which has not been reported before. It is similar to the canonical ensemble in which the phase transition only takes place when $k=-1$. But it also has its unique characteristics that the location of the phase transition point depends on the value of potential, which is different from the canonical ensemble where the phase transition point is independent of the parameters. After an analytical check of Ehrenfest scheme, we find that the new phase transition is a second order one. It is also found that the thermodynamics of the black hole in HoravaLifshitz gravity is quite different from that in Einstein gravity.
\end{abstract}

\footnotetext{
${ }^{1}$ mojiexiong@gmail.com

${ }^{2}$ Corresponding author:wbliu@bnu.edu.cn
} 


\section{Contents}

$\begin{array}{llr}1 & \text { Introduction } & 1\end{array}$

2 Review of thermodynamics of the charged topological black hole $\quad 2$

3 Phase transition and geometrothermodynamics in fixed-charge ensemble 4

4 Phase transition and geometrothermodynamics in fixed-potential ensemble

5 Analytical check of Ehrenfest equations in the fixed-potential ensemble 15

6 Conclusions

\section{Introduction}

Black hole thermodynamics has become a fascinating topic in the theoretical physics since Bekenstein and Hawking investigated the black hole entropy and identified black holes as thermodynamic objects [1], [2].Various thermodynamic properties of black holes have been studied widely including the phase transition. In 1983, Hawking and Page discovered that there exists a phase transition between the Schwarzschild AdS black hole and the thermal AdS space [3]. From then on, phase transitions of black holes have aroused theoretical physicists' attention and have been intensely investigated from different perspectives [4][37].

On the one hand, traditional thermodynamics has been applied to investigate the critical behavior of a black hole. For example, utilizing Clausius-Clapeyron-Ehrenfests equations, one can classify phase transitions as first order or higher order transitions. For a first order transition the Clausius-Clapeyron equation is satisfied. While for a second order transition Ehrenfests relations are satisfied. Recently, Banerjee et al. developed a scheme based on Ehrenfests relations to study phase transitions in black holes [24]-[29]. They considered the black holes as grand-canonical ensembles and performed a detailed analysis of Ehrenfests relations using both analytical and graphical techniques.

On the other hand, geometric approach has served as an alternative way to study phase transitions of black holes. Riemannian geometry in the space of equilibrium states was introduced by Weinhold [38] and Ruppeiner [39]. Weinhold proposed metric structure in the energy representation as $g_{i, j}^{W}=\partial_{i} \partial_{j} M\left(U, N^{a}\right)$, which was the Hessian matrix of the internal energy $U$ with respect to the extensive thermodynamic variables $N^{a}$. Ruppeiner defined metric structure as $g_{i, j}^{R}=-\partial_{i} \partial_{j} S\left(U, N^{a}\right)$, which was the Hessian of the entropy. However, Weinhold's and Ruppeiner's metrics are not invariant under Legendre transformations and 
sometimes lead to contradictory results [40]-[41]. Taking Legendre invariance into consideration, Quevedo et al. [42] recently presented a new formalism of geometrothermodynamics, which allows us to derive Legendre invariant metrics in the space of equilibrium states. Geometrothermodynamics presents a unified geometry where the metric structure can give a well description of various types of black hole thermodynamics [43]-[51].

Here, we would like to focus our attention on the phase transition of the charged topological black hole in Hořava-Lifshitz gravity. Hořava-Lifshitz (HL) theory is a nonrelativistic renormalizable theory of gravity at a Lifshitz point proposed by Hořava [52][54]. HL gravity provides a fascinating framework for one to explore the connections between ordinary gravity and string theory. The black hole solutions [55]-[59] and thermodynamic properties [60]-[68] have attracted a lot of attention. Concerning the phase transition, some efforts have also been made. Koutsoumbas et al. [17] mainly discussed the perturbative behaviour and quasi-normal modes of charged topological AdS black holes. Cao et al. [21] studied black hole phase transitions in (deformed) HL gravity, including the charged/uncharged topological black holes and Kehagias-Sfetsos (KS) black hole. However, their geometric approach was based on the Ruppeiner and Weinhold metrics and failed to predict the phase transition. Quevedo et al. [22]-[23] investigated the geometrothermodynamics in HL gravity. But it mainly handled the Cai-Cao-Ohta (CCO) topological black holes and left the charged topological black holes uninvestigated. Wei et al. [30] mainly discussed the thermodynamic geometry and phase transition of KS black hole in the deformed HL gravity while Majhi et al. [31] focused their attention on the scaling behavior of topological charged black holes in HL gravity. In this paper, we would like to further elaborate the research on the phase transition of the charged topological black hole in HL gravity. Aiming at a unified picture, the phase transition would be considered not only in canonical ensemble with the fixed charge case but also in grand-canonical ensemble with the fixed potential case. Both geometrothermodynamics and the Ehrenfests scheme would be applied to carry out the research.

The organization of our paper is as follows. In Section 2, the thermodynamics of the charged topological black hole in HL gravity will be reviewed briefly. In Section 3, the charged topological black hole as canonical ensemble will be investigated in geometrothermodynamics. In Section 4, the phase transition will be studied taking the black hole as grand-canonical ensemble. To investigate the nature of the new phase transition in Section 4, an analytical check of Ehrenfest equations will be carried out in Section 5. In the end, a discussion is given in Section 6 .

\section{Review of thermodynamics of the charged topological black hole}

The charged topological black hole solution in Hořava-Lifshitz gravity has been discussed in [56]. For simplicity, the dynamical coupling constant $\lambda$ can be set to one. And the 
metric is given as

$$
\begin{aligned}
& d s^{2}=-\tilde{N}(r)^{2} d t^{2}+\frac{d r^{2}}{f(r)}+r^{2} d \Omega_{k}^{2}, \\
& f(r)=k+x^{2}-\sqrt{c_{0} x-\frac{q^{2}}{2}}, \quad x=\sqrt{-\Lambda} r,
\end{aligned}
$$

where $\Lambda$ corresponds to the negative cosmological constant and $d \Omega_{k}$ is the line element of a two dimensional Einstein space with constant scalar curvature $2 k$. Without loss of generality, one can take $k=0, \pm 1$ respectively. For the metric given above, $\widetilde{N}=N_{0}$ could be set to one. Solving the equation $f(r)=0$, we can get the largest positive root, from which we can determine the event horizon radius. Denoting $l^{2}=-\frac{1}{\Lambda}$, the relevant quantities have been reviewed in [21] as

$$
\begin{aligned}
T & =\frac{6 x_{+}^{4}+4 k x_{+}^{2}-2 k^{2}-q^{2}}{16 k l^{2} \pi x_{+}+16 l^{2} \pi x_{+}^{3}}, \\
S & =\frac{\pi \kappa^{2} \mu^{2} \Omega_{k}}{4}\left(x_{+}^{2}+2 k \ln \left(x_{+}\right)\right)+S_{0}, \\
\Phi & =\frac{q}{x_{+}}+\Phi_{0}, \\
Q & =\frac{\kappa^{2} \mu^{2} \Omega_{k}}{16 l^{2}} q, \\
M & =\frac{\kappa^{2} \mu^{2} \Omega_{k}}{16 l^{2}} c_{0},
\end{aligned}
$$

where $c_{0}=\frac{2 k^{2}+q^{2}+4 k x_{+}^{2}+2 x_{+}^{4}}{2 x_{+}}, c_{0}, q$ are the integration constants and $\kappa, \mu$ are the constant parameters of the theory. $\Omega_{k}$ is the volume of two dimensional Einstein space.

From Eq.(2.3), we can easily find that the charged topological black hole becomes an extremal one when $q^{2}=6 x_{+}^{4}+4 k x_{+}^{2}-2 k^{2}$. Hawking temperature will be negative when $6 x_{+}^{4}+4 k x_{+}^{2}-2 k^{2}<q^{2}$, which implies the existence of some unphysical regions. To show the variation of Hawking temperature explicitly, we plot Figure 1 using Eq.(2.3). In Figure 1, we exhibit the temperature $T$ vs. $x_{+}$respectively for the cases $k=0, \pm 1$ (Note that parameters are chosen as $c=l=G=\kappa=\mu=\Omega_{k}=1$ in all figures in this paper.)

From Figure 1, we can find that each case has some unphysical regions with negative Hawking temperature. For the case $k=0,1$, Hawking temperature increases monotonically. However, things are different for the case $k=-1 . x_{+}=1$ divides the region into two parts. When $x_{+}>1$, Hawking temperature increases monotonically. When $0<x_{+}<1$, there exists a minimum Hawking temperature, which can be derived from

$$
\frac{\partial T_{k=-1}}{\partial x_{+}}=\frac{\partial}{\partial x_{+}}\left(\frac{6 x_{+}^{4}-4 x_{+}^{2}-2-q^{2}}{-16 l^{2} \pi x_{+}+16 l^{2} \pi x_{+}^{3}}\right)=0 .
$$

Solving Eq.(2.8), we can get the corresponding $x_{+}$and the minimum Hawking temperature as

$$
x_{+}=\frac{\sqrt{3}}{3}, \quad T_{\min }=\frac{\sqrt{3}\left(8+3 q^{2}\right)}{32 \pi l^{2}} .
$$

Note that the location of $x_{+}$that corresponds to the minimum Hawking temperature is independent of the charge parameter $q$. 


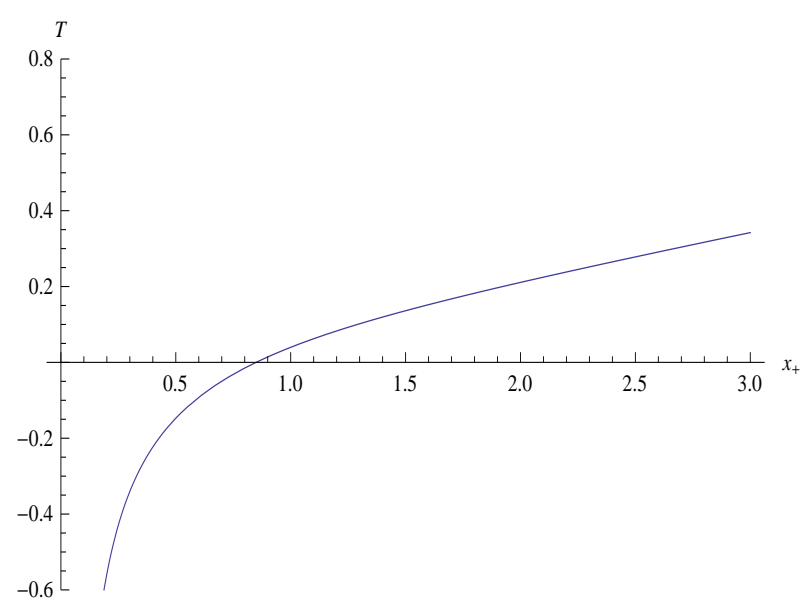

(a)

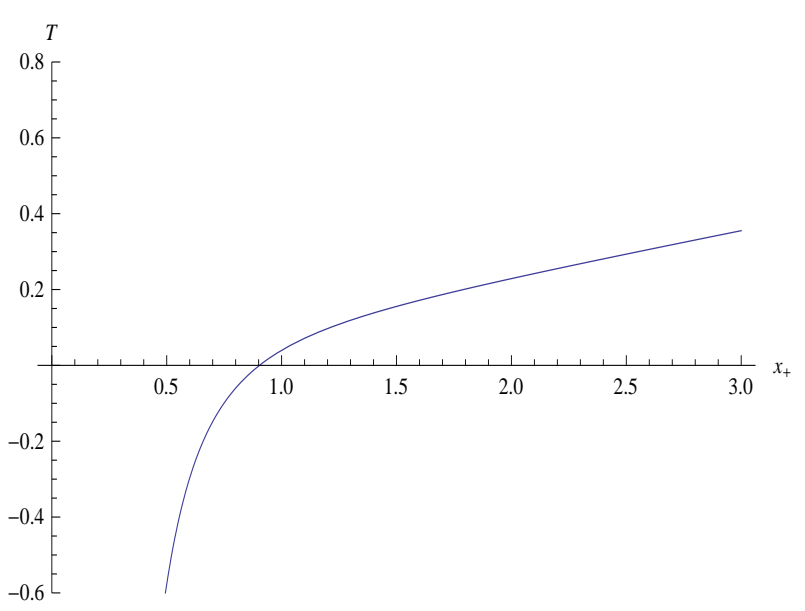

(b)

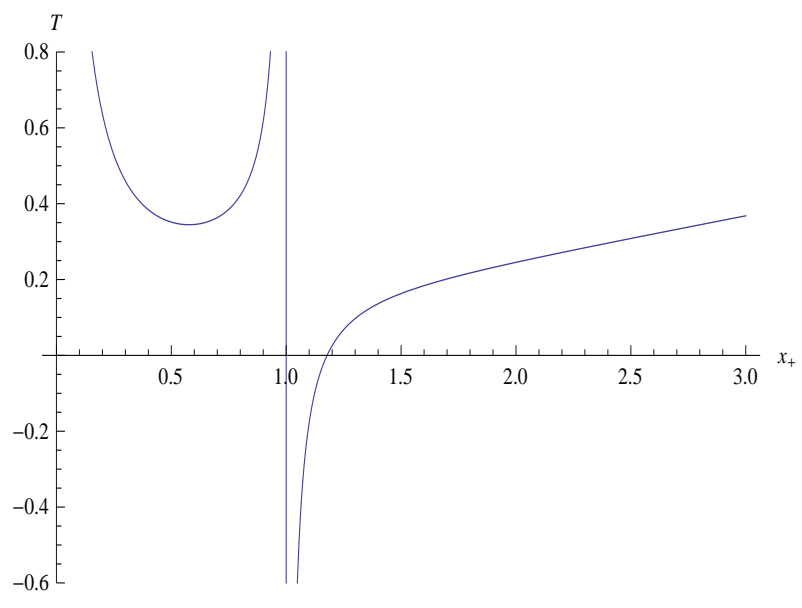

(c)

Figure 1. Hawking temperature $T$ vs. $x_{+}$for $(\mathrm{a}) k=1, q=2$ (b) $k=0, q=2$ (c) $k=-1, q=2$

\section{Phase transition and geometrothermodynamics in fixed-charge ensem- ble}

When the charge of black hole is fixed, the specific heat can be given as

$$
C_{Q}=T\left(\frac{\partial S}{\partial T}\right)_{Q}=\frac{-\pi\left(k+x_{+}^{2}\right)^{2}\left[q^{2}+2\left(k-3 x_{+}^{2}\right)\left(k+x_{+}^{2}\right)\right] \kappa^{2} \Omega_{k} \mu^{2}}{2\left(k+3 x_{+}^{2}\right)\left[q^{2}+2\left(k+x_{+}^{2}\right)^{2}\right]} .
$$

From Eq.(3.1), we can conclude that $C_{Q}$ diverges when $k+3 x_{+}^{2}=0$. The equation $k+3 x_{+}^{2}=0$ has positive root only when $k=-1$. And the root can be solved as $x_{+}=$ $\frac{\sqrt{3}}{3}$. It is quite interesting to note that the point where $C_{Q}$ diverges is independent of charge parameter $q$ and coincides with the point corresponding to the minimum Hawking temperature.

To observe the possible divergence of $C_{Q}$, we plot Figure 2 using Eq.(3.1). In Figure 2, we exhibit the behavior of $C_{Q}$ respectively for the cases $k=0, \pm 1$. We can see that $C_{Q}$ is 


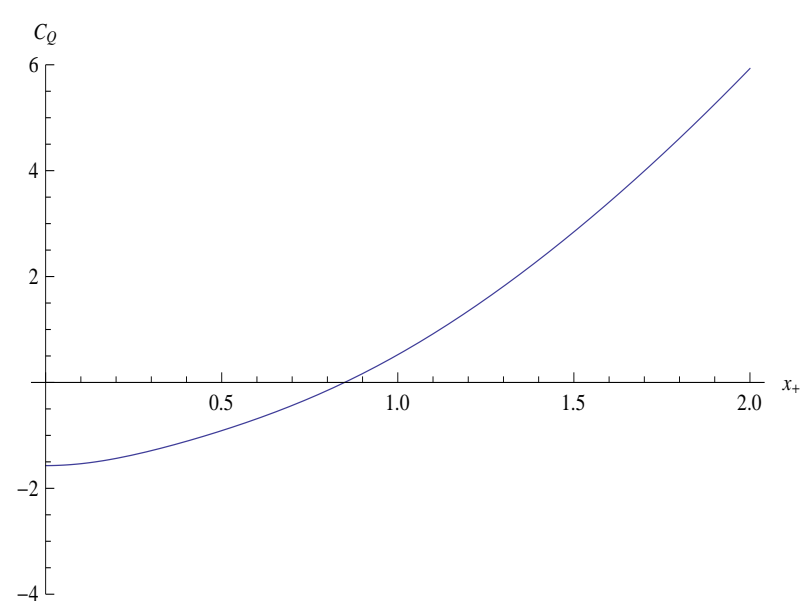

(a)

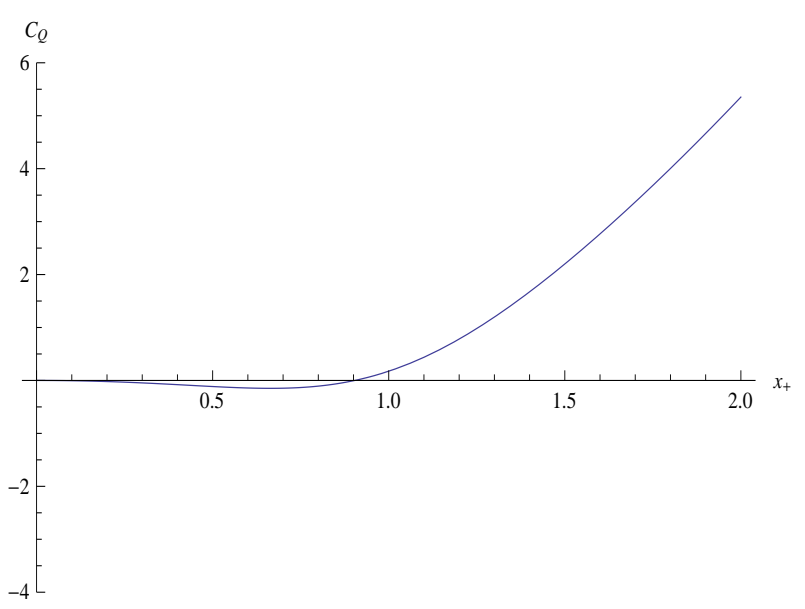

(b)

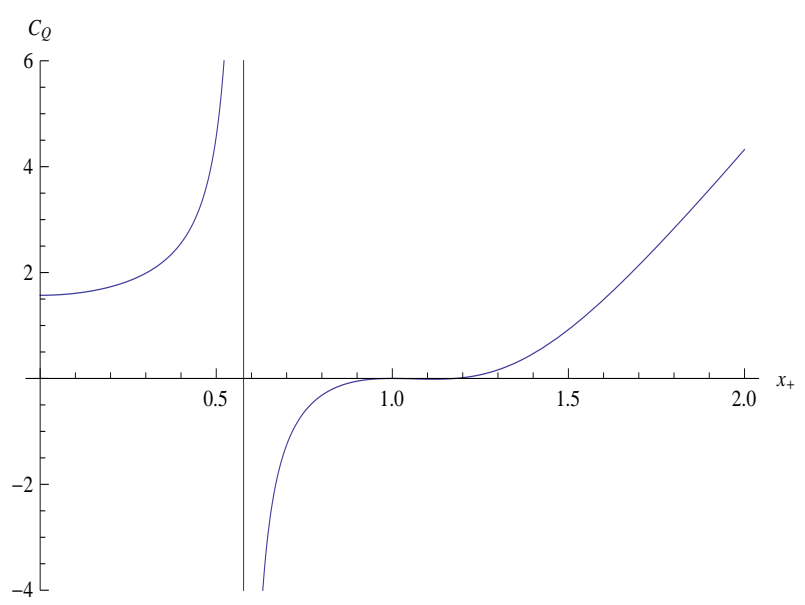

(c)

Figure 2. $C_{Q}$ vs. $x_{+}$for (a) $k=1, q=2$ (b) $k=0, q=2$ (c) $k=-1, q=2$

continuous for the cases $k=0,1$ and no phase transition takes place. However, the curve for the case $k=-1$ gives an infinite discontinuity, which suggests the existence of phase transition. The phase transition location is $x_{+}=\frac{\sqrt{3}}{3}$.

To confirm the phase transition in canonical ensemble, we would like to perform an analysis of the behavior of free energy. The free energy which is defined by $F=M-T S$ can be obtained as

$$
\begin{aligned}
F= & \frac{\kappa^{2} \mu^{2} \Omega_{k}}{64 l^{2} x_{+}\left(k+x_{+}^{2}\right)} \times\left[4 k^{3}+14 k^{2} x_{+}^{2}+8 k x_{+}^{4}-2 x_{+}^{6}+2 k q^{2}+3 q^{2} x_{+}^{2}\right. \\
& \left.+k \ln x_{+}\left(4 k^{2}+2 q^{2}-8 k x_{+}^{2}-12 x_{+}^{4}\right)\right] .
\end{aligned}
$$

Figure 3 shows the free energy vs. the temperature for three different cases. For $k=1,0$, the free energy decreases steadily when the temperature increases. However, for $k=-1$, two different phases are described by two wings which are joined at the point where the free energy reaches a maximum value. According to traditional thermodynamics, the system is 


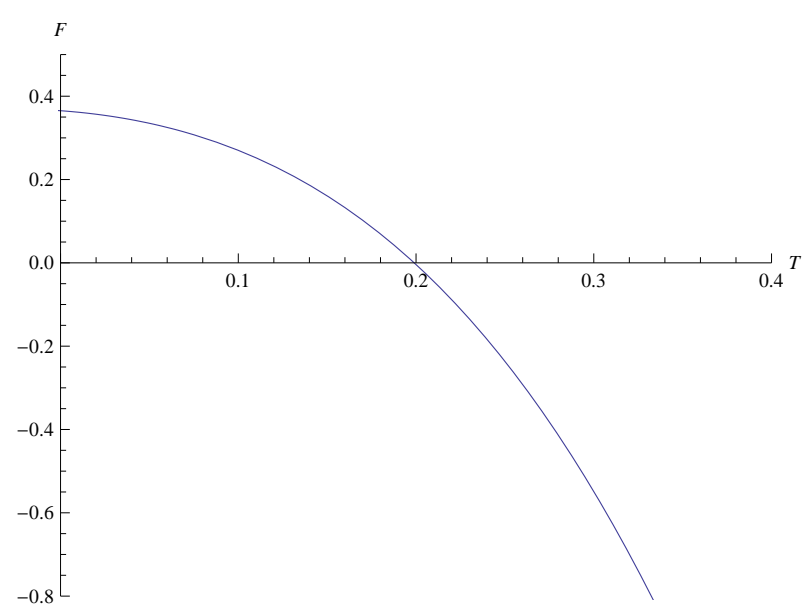

(a)

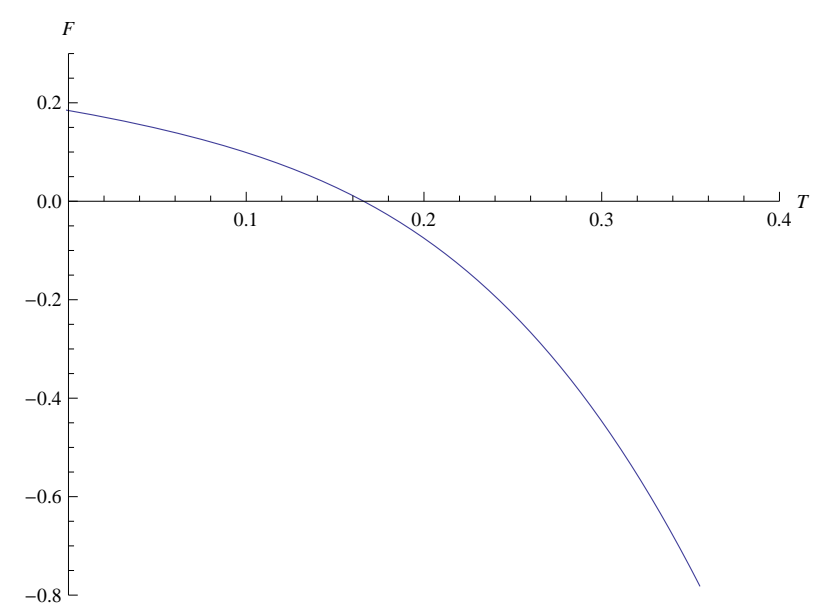

(b)

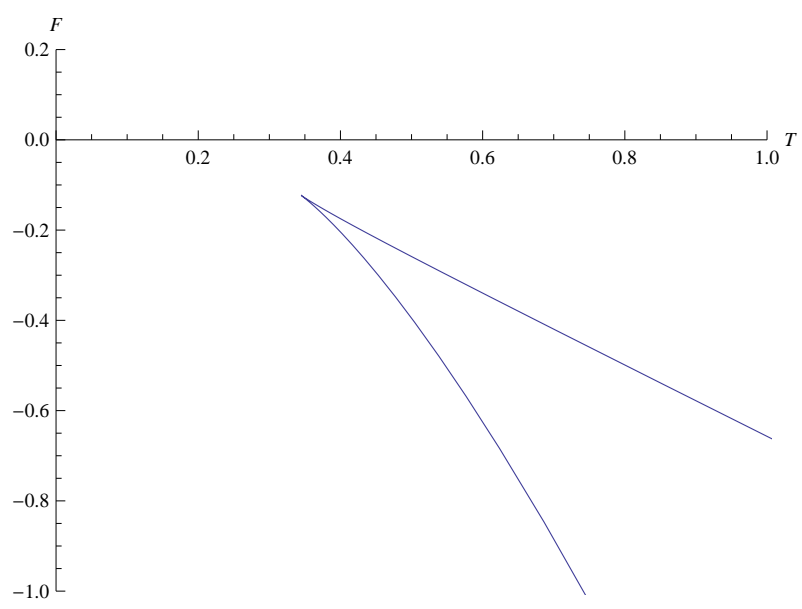

(c)

Figure 3. $F$ vs. $T$ for (a) $k=1, q=2$ (b) $k=0, q=2$ (c) $k=-1, q=2$

most unstable at this point and would eventually passes to the locally stable phase which corresponds to the lower wing. It is quite interesting to note that the point of the maximum free energy also corresponds to the minimum Hawking temperature. As we stated before, it is also the point where the local phase transition takes place from a locally unstable small black hole to a locally stable large black hole. So we can conclude that the local phase transition points not only witness the divergence of the specific heat but also witness the minimum temperature and maximum free energy.

Before we go further to investigate its geometrothermodynamics, we would like to briefly review the construction of geometrothermodynamics. According to Ref. [42], the $(2 n+1)$-dimensional thermodynamic phase space $\mathcal{T}$ can be coordinated by the set of independent quantities $\left\{\phi, E^{a}, I^{a}\right\}$, where $a=1, \cdots, n$, the positive integer $n$ represents the number of thermodynamic degree of freedom of the system, $\phi$ corresponds to the thermodynamic potential, and $E^{a}, I^{a}$ are the extensive and intensive thermodynamic variables respectively. The fundamental Gibbs 1- form defined on $\mathcal{T}$ can then be written as 
$\Theta=d \phi-\delta_{a b} I^{a} d E^{b}$, where $\delta_{a b}=\operatorname{diag}(1, \cdots, 1)$. Considering a non-degenerate Riemannian metric $G$, a contact Riemannian manifold can be defined from the set $(\mathcal{T}, \Theta, G)$ if the condition $\Theta \wedge(\Theta)^{n} \neq 0$ is satisfied. Legendre transformation is such a special case of contact transformation that it can keep the contact structure of $\mathcal{T}$ invariant. Legendre invariance guarantees that the geometric properties of $G$ do not depend on the thermodynamic potential. Utilizing a smooth map $\varphi: \varepsilon \rightarrow \mathcal{T}$, i.e. $\varphi:\left(E^{a}\right) \mapsto\left(\phi, E^{a}, I^{a}\right)$, a submanifold $\varepsilon$ called the space of thermodynamic equilibrium states can be induced. $\phi=\phi\left(E^{a}\right)$ and the map satisfies the condition $\varphi^{*}(\Theta)=\varphi^{*}\left(d \phi-\delta_{a b} I^{a} d E^{b}\right)=0$, where $\varphi^{*}$ is the pullback of $\varphi$. $\varepsilon \subset \mathcal{T}$ and $\varepsilon$ is an $n$-dimensional Riemannian submanifold. Furthermore, a thermodynamic metric $g$ can be induced in a canonical manner in the equilibrium manifold $\varepsilon$ by the smooth $\operatorname{map} \varphi$.

As proposed by Quevedo, the non-degenerate metric $G$ and the thermodynamic metric $g$ can be written as follows [49]

$$
\begin{aligned}
& G=\left(d \phi-\delta_{a b} I^{a} d E^{b}\right)^{2}+\left(\delta_{a b} E^{a} I^{b}\right)\left(\eta_{c d} d E^{c} d I^{d}\right) \\
& g=\varphi^{*}(G)=\left(E^{c} \frac{\partial \phi}{\partial E^{c}}\right)\left(\eta_{a b} \delta^{b c} \frac{\partial^{2} \phi}{\partial E^{c} \partial E^{d}} d E^{a} d E^{d}\right),
\end{aligned}
$$

where $\eta_{a b}=\operatorname{diag}(-1, \cdots, 1)$.

Now let's begin to apply geometrothermodynamics to investigate the phase transition of the charged topological black hole in HL gravity. To construct geometrothermodynamics in fixed-charge ensemble, we choose $M$ as the thermodynamic potential, and $S, Q$ as extensive variables. The corresponding thermodynamic phase space is a 5 -dimensional one coordinated by the set of independent coordinates $\{M, S, Q, T, \Phi\}$. The fundamental Gibbs 1- form defined on $\mathcal{T}$ can be written as

$$
\Theta=d M-T d S-\Phi d Q
$$

and the non-degenerate metric $G$ from Eq.(3.3) is

$$
G=(d M-T d S-\Phi d Q)^{2}+(T S+\Phi Q)(-d S d T+d Q d \Phi)
$$

Introducing the map

$$
\varphi:\{S, Q\} \mapsto\left\{M(S, Q), S, Q, \frac{\partial M}{\partial S}, \frac{\partial M}{\partial Q}\right\}
$$

the space of thermodynamic equilibrium states can be induced. According to Eq.(3.4), the thermodynamic metric $g$ can be written as follows

$$
g=\left(S \frac{\partial M}{\partial S}+Q \frac{\partial M}{\partial Q}\right)\left(-\frac{\partial^{2} M}{\partial S^{2}} d S^{2}+\frac{\partial^{2} M}{\partial Q^{2}} d Q^{2}\right)
$$


Utilizing Eqs.(2.4),(2.6)and (2.7), we can easily calculate the relevant quantities in Eq.(3.8) as below

$$
\begin{aligned}
\frac{\partial M}{\partial S} & =\frac{-q^{2}-2\left(k-3 x_{+}^{2}\right)\left(k+x_{+}^{2}\right)}{16 l^{2} \pi x_{+}\left(k+x_{+}^{2}\right)} \\
\frac{\partial M}{\partial Q} & =\frac{q}{x_{+}} \\
\frac{\partial^{2} M}{\partial S^{2}} & =\frac{\left(k+3 x_{+}^{2}\right)\left[q^{2}+2\left(k+x_{+}^{2}\right)^{2}\right]}{8 l^{2} \pi^{2} x_{+}\left(k+x_{+}^{2}\right)^{2} \kappa^{2} \mu^{2} \Omega_{k}} \\
\frac{\partial^{2} M}{\partial Q^{2}} & =\frac{16 l^{2}}{\kappa^{2} \mu^{2} \Omega_{k} x_{+}} .
\end{aligned}
$$

Comparing Eqs.(3.9),(3.10) with Eqs.(2.3),(2.5), we find

$$
\frac{\partial M}{\partial S}=T, \quad \frac{\partial M}{\partial Q}=\Phi-\Phi_{0}
$$

To assure that the first law of black hole thermodynamics holds,we can derive from Eq.(3.13) $\Phi_{0}=0$. Substituting Eqs.(3.9)-(3.12) into Eq.(3.8), we can calculate the component of the thermodynamic metric $g$ as

$$
\begin{aligned}
g_{Q Q} & =\frac{q^{2}}{x_{+}^{2}}-\frac{\left[q^{2}+2\left(k+x_{+}^{2}\right)\left(k-3 x_{+}^{2}\right)\right]\left[4 S_{0}+\pi \kappa^{2} \mu^{2} \Omega_{k}\left(x_{+}^{2}+2 k \ln x_{+}\right)\right]}{4 \pi x_{+}^{2}\left(k+x_{+}^{2}\right) \kappa^{2} \mu^{2} \Omega_{k}}, \\
g_{S S} & =-\frac{A\left(x_{+}, q\right)}{512 l^{4} \pi^{3} \kappa^{2} \mu^{2} \Omega_{k} x_{+}^{2}\left(k+x_{+}^{2}\right)^{4}},
\end{aligned}
$$

where

$$
\begin{aligned}
A\left(x_{+}, q\right)= & \left(k+3 x_{+}^{2}\right)\left[q^{2}+2\left(k+x_{+}^{2}\right)^{2}\right] \\
& \times\left\{-\left[q^{2}+2\left(k-3 x_{+}^{2}\right)\left(k+x_{+}^{2}\right)\right]\left[4 S_{0}+\pi \kappa^{2} \mu^{2} \Omega_{k}\left(x_{+}^{2}+2 k \ln x_{+}\right)\right]\right. \\
& \left.+4 q^{2} \pi \kappa^{2} \mu^{2} \Omega_{k}\left(k+x_{+}^{2}\right)\right\} .
\end{aligned}
$$

Till now, we can obtain the Legendre invariant scalar curvature as

$$
\mathfrak{R}_{Q}=\frac{B\left(x_{+}, Q\right)}{D\left(x_{+}, Q\right)}
$$

where

$$
\begin{aligned}
D\left(x_{+}, Q\right)= & \left(k+3 x_{+}^{2}\right)^{2} \times\left(128 l^{4} Q^{2}+\kappa^{4} \mu^{4} \Omega_{k}^{2}\left(k+x_{+}^{2}\right)^{2}\right)^{2} \\
& \times\left\{512 S_{0} l^{4} Q^{2}-128 \pi l^{4} Q^{2} \kappa^{2} \mu^{2} \Omega_{k}\left(4 k+3 x_{+}^{2}\right)\right. \\
& +4 S_{0} \kappa^{4} \mu^{4} \Omega_{k}^{2}\left(k-3 x_{+}^{2}\right)\left(k+x_{+}^{2}\right)+\pi \kappa^{6} \mu^{6} \Omega_{k}^{3} x_{+}^{2}\left(k-3 x_{+}^{2}\right)\left(k+x_{+}^{2}\right) \\
& \left.+2 k \pi \kappa^{2} \mu^{2} \Omega_{k}\left[128 l^{4} Q^{2}+\kappa^{4} \mu^{4} \Omega_{k}^{2}\left(k-3 x_{+}^{2}\right)\left(k+x_{+}^{2}\right)\right] \ln x_{+}\right\}^{3} .
\end{aligned}
$$

From Eq.(3.18), we can find that the Legendre invariant scalar curvature diverges when $k+3 x_{+}^{2}=0$, which corresponds to $k=-1, x_{+}=\frac{\sqrt{3}}{3}$. That is the exact point where the phase transition takes place. To get an intuitive sense on this issue, we plot Figure 4, which shows the correpondence of the divergence of specific heat $C_{Q}$ and the thermodynamic scalar curvature of $\mathfrak{R}_{Q}$. From Figure 4, we learn that the Legendre invariant metric constructed in geometrothermodynamics correctly produces the behavior of the thermodynamic interaction and phase transition structure. 


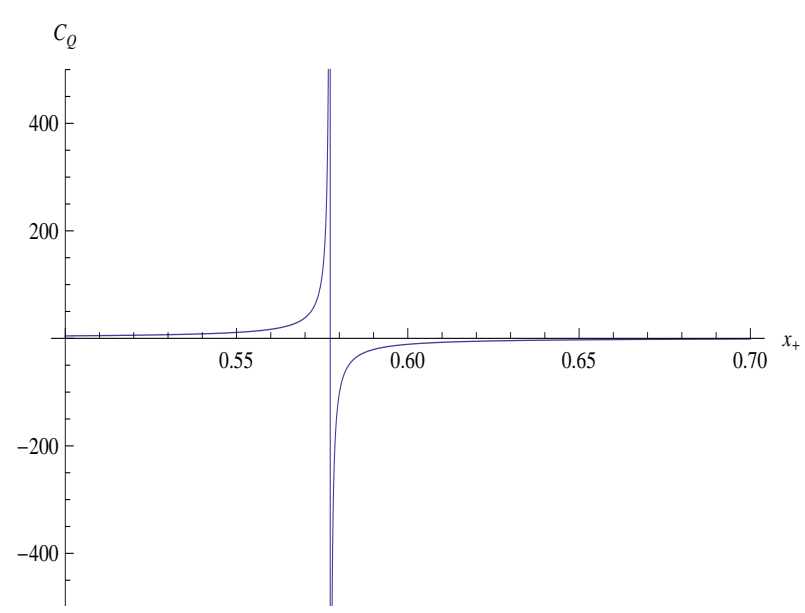

(a)

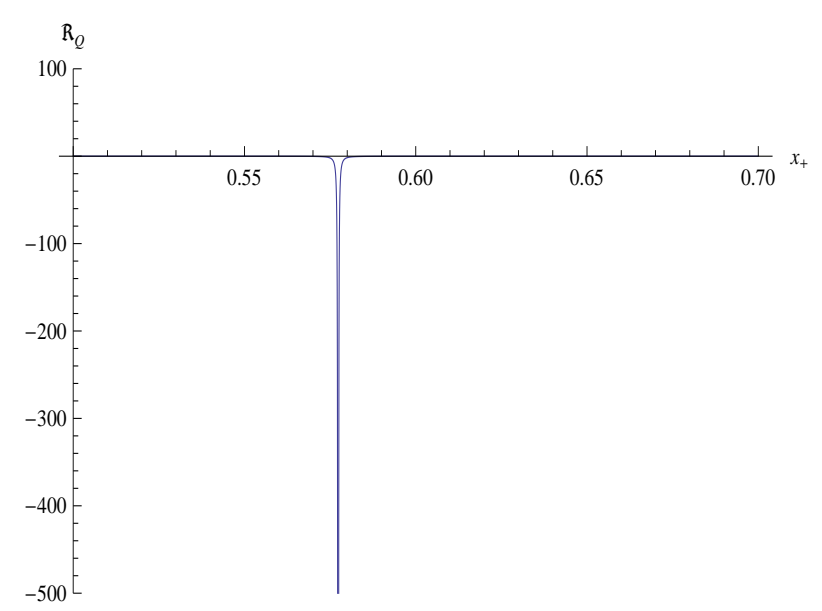

(b)

Figure 4. (a) $C_{Q}$ vs. $x_{+}$for $k=-1, Q=2(b) \mathfrak{R}_{Q}$ vs. $x_{+}$for $k=-1, Q=2$

\section{Phase transition and geometrothermodynamics in fixed-potential en- semble}

When the potential of black hole is fixed, the specific heat is shown as

$$
C_{\Phi}=T\left(\frac{\partial S}{\partial T}\right)_{\Phi}=\frac{-\pi \kappa^{2} \mu^{2} \Omega_{k}\left(k+x_{+}^{2}\right)^{2}\left(2 k^{2}-4 k x_{+}^{2}-6 x_{+}^{4}+x_{+}^{2} \Phi^{2}\right)}{2\left(2 k^{3}+10 k^{2} x_{+}^{2}+14 k x_{+}^{4}-k x_{+}^{2} \Phi^{2}+x_{+}^{4} \Phi^{2}+6 x_{+}^{6}\right)} .
$$

Apparently, $C_{\Phi}$ may diverge when

$$
2 k^{3}+10 k^{2} x_{+}^{2}+14 k x_{+}^{4}-k x_{+}^{2} \Phi^{2}+x_{+}^{4} \Phi^{2}+6 x_{+}^{6}=0,
$$

which suggests a possible phase transition. However, the phase transition point characterized by Eq.(4.2) is not intuitive. To gain an intuitive understanding, we plot Figure 5 using Eq.(4.1) . In Figure 5, we exhibit the behavior of $C_{\Phi}$ respectively for the cases $k=0, \pm 1$. We can find that $C_{\Phi}$ diverges only for $k=-1$, which is similar to the fixed-charged ensemble. To check whether the phase transition point locates in the physical region, we also plot the Hawking temperature $T$ vs. $x_{+}$for the case $k=-1$ in Figure 5(c). It is shown that the phase transition point locates in the positive temperature region. Figure $5(\mathrm{c})$ can be divided into two phases. One is thermodynamically stable $\left(C_{\Phi}>0\right)$ with small radius while the other is unstable $\left(C_{\Phi}<0\right)$ with large radius. So the phase transition takes place between small black hole and large black hole.

To confirm the phase transition in grand-canonical ensemble, we would like to perform an analysis of the behavior of the Gibbs potential. The Gibbs potential which is defined by $G=M-T S-\Phi Q$ can be obtained as

$$
\begin{aligned}
G= & \frac{\kappa^{2} \mu^{2} \Omega_{k}}{64 l^{2} x_{+}\left(k+x_{+}^{2}\right)} \times\left[2\left(k+x_{+}^{2}\right)\left(2 k^{2}+5 k x_{+}^{2}-x_{+}^{4}\right)\right. \\
& \left.+2 k \ln x_{+}\left(2 k^{2}+x_{+}^{2} \Phi^{2}-4 k x_{+}^{2}-6 x_{+}^{4}\right)-x_{+}^{2}\left(2 k+x_{+}^{2}\right) \Phi^{2}\right] .
\end{aligned}
$$




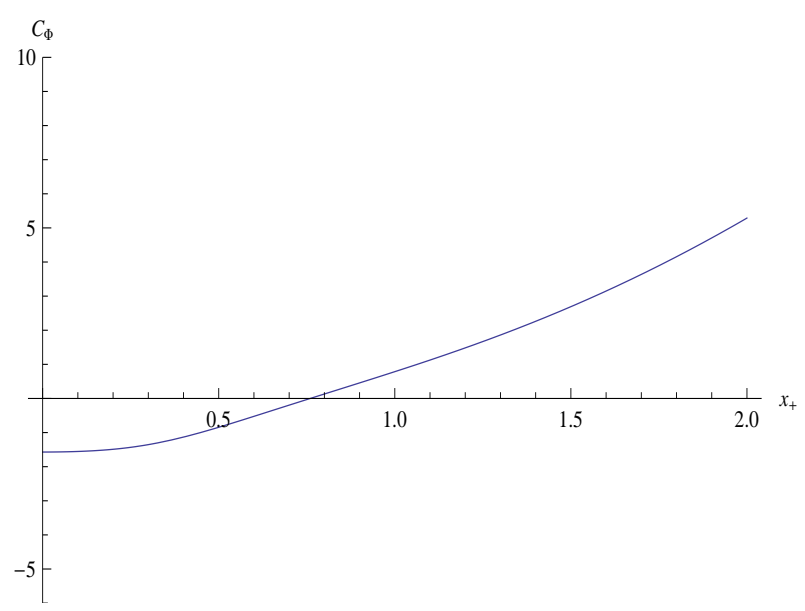

(a)

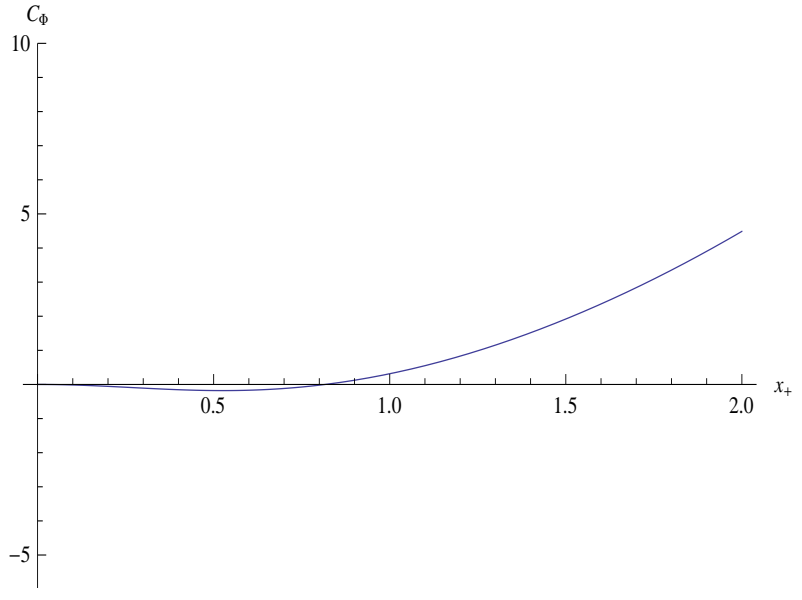

(b)

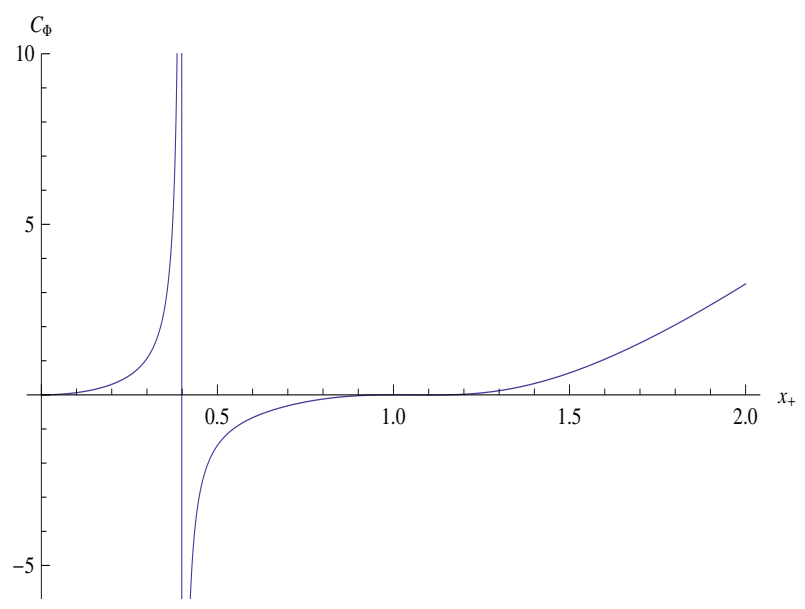

(c)

Figure 5. $C_{\Phi}$ vs. $x_{+}$for (a) $k=1, \Phi=2$ (b) $k=0, \Phi=2$ (c) $k=-1, \Phi=2$

Figure 6 shows the Gibbs potential vs. the temperature for three different cases. For $k=1,0$, the Gibbs potential decreases with the increasing of the Hawking temperature. For $k=-1$, two wings are connected at the point where Gibbs potential reaches a maximum value. According to traditional thermodynamics, the system is most unstable at this point and would eventually passes to the locally stable phase which has the low Gibbs potential corresponding to the lower wing.

According to Eq.(4.2), the phase transition point may depend on the value of the potential $\Phi$. To trace the variation of the phase transition point due to potential, we plot Figure 7 with different potential values. In Figure 7, we can see that the phase transition point changes with potential $\Phi$. When $\Phi$ increases, the value of $x_{+}$corresponding to the phase transition point tends to decrease. Figure 8 which shows the behavior of the Hawking temperature with different choices of potential values indicates that the phase transition points all locate in the physical regions. With the increasing of the potential, the minimum temperature tends to increase while the value of $x_{+}$corresponding to the minimum Hawking 


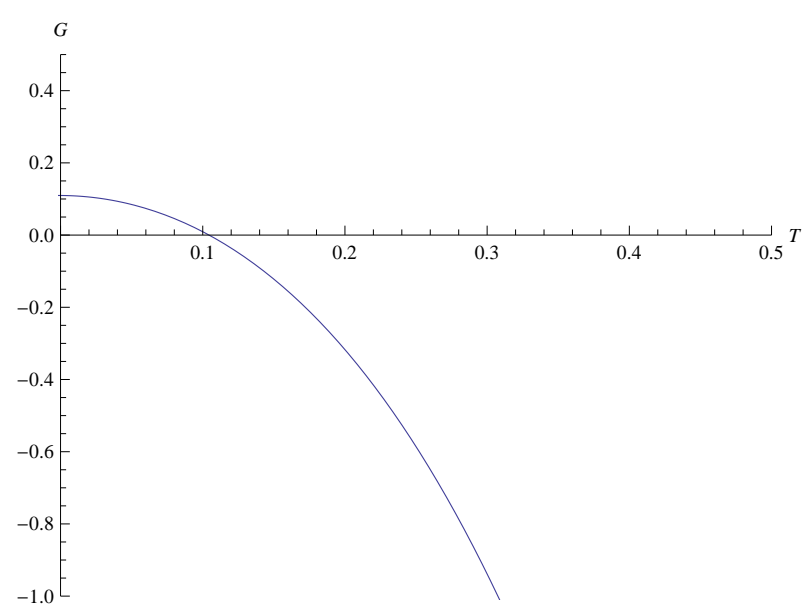

(a)

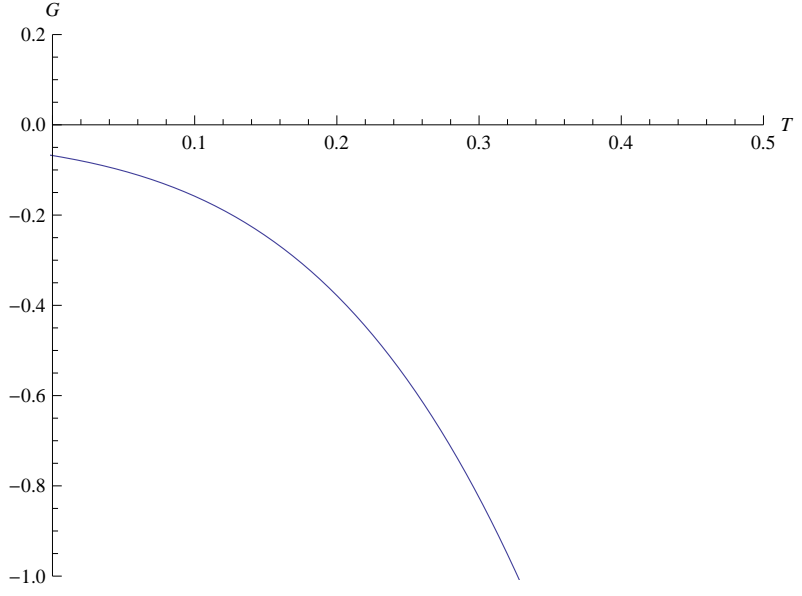

(b)

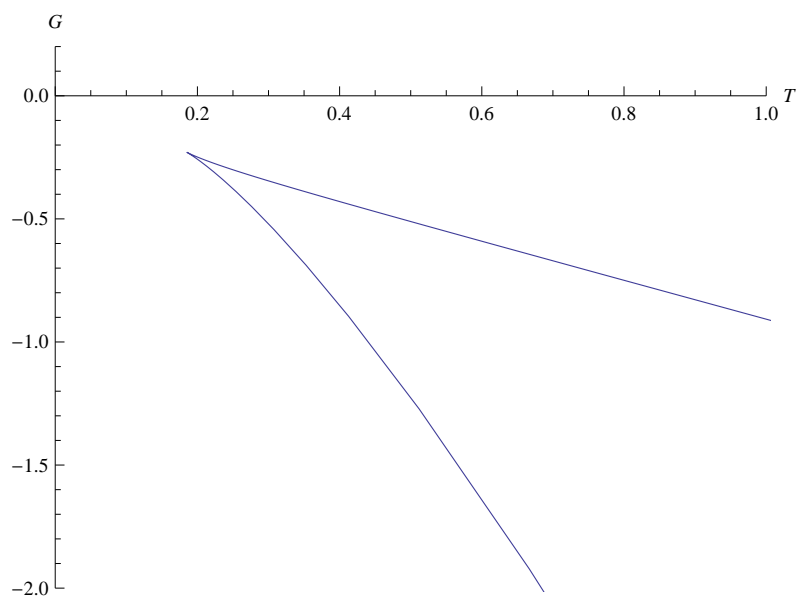

(c)

Figure 6. $G$ vs. $T$ for (a) $k=1, \Phi=2$ (b) $k=0, \Phi=2$ (c) $k=-1, \Phi=2$

temperature tends to decrease. It is quite interesting to note from Figure 9 that the point having the maximum Gibbs potential also corresponds to the minimum Hawking temperature. With the increasing of the potential, the minimum temperature tends to increase, which is consistent with Figure 8. Finally, we can draw the conclusion that the local phase transition points not only witness the divergence of the specific heat but also witness the minimum temperature and maximum Gibbs potential.

The phase transition in the grand-canonical ensemble has never been reported before. It is similar to the canonical ensemble in which the phase transition only takes place for $k=-1$. It is very interesting that the location of the phase transition point changes with potential, which is different from canonical ensemble where the phase transition point is independent of the parameters.

To construct geometrothermodynamics in fixed-potential ensemble, we define the thermodynamic potential as [69]

$$
J_{\Phi}=M-\Phi Q
$$




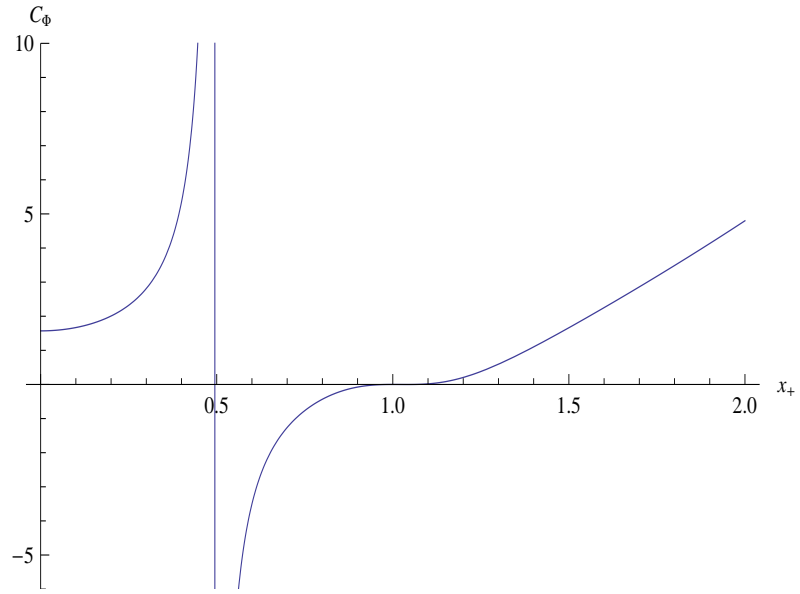

(a)

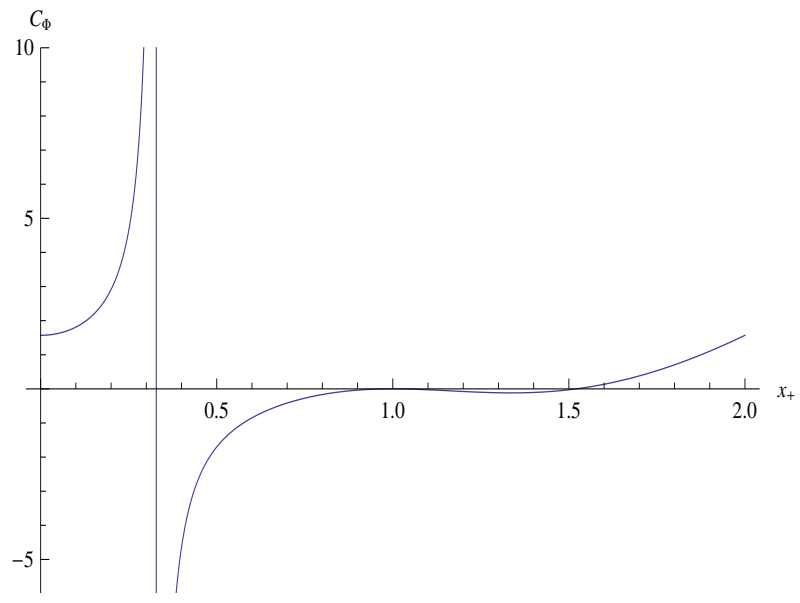

(c)

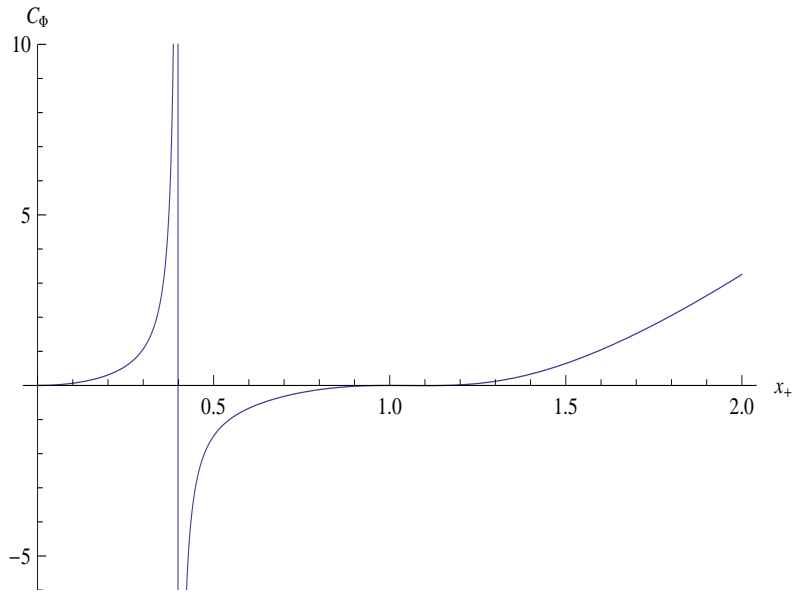

(b)

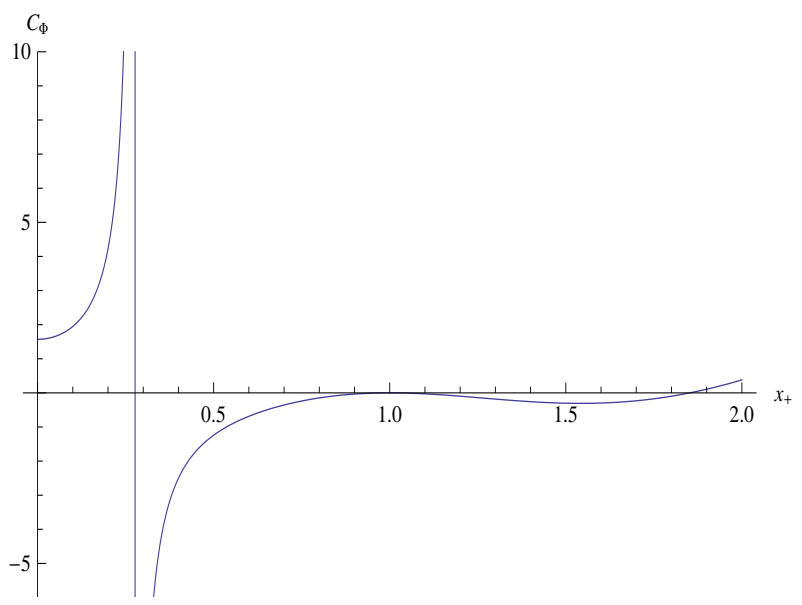

(d)

Figure 7. $C_{\Phi}$ vs. $x_{+}$for $(\mathrm{a}) k=-1, \Phi=1$ (b) $k=-1, \Phi=2$ (c) $k=-1, \Phi=3$ (d) $k=-1, \Phi=4$

Substituting Eqs.(2.5)-(2.7) into Eq.(4.4), we can obtain the explicit form of $J_{\Phi}$ as

$$
J_{\Phi}=\frac{\kappa^{2} \mu^{2} \Omega_{k}\left[2\left(k+x_{+}^{2}\right)^{2}-\left(\Phi^{2}-\Phi_{0}^{2}\right) x_{+}^{2}\right]}{32 l^{2} x_{+}} .
$$

It is easy to conclude from Eq.(4.4) that

$$
d J_{\Phi}=d M-(\Phi d Q+Q d \Phi)=T d S-Q d \Phi .
$$

Unlike the fixed-charge ensemble, $S, \Phi$ are taken as extensive variables here. And the corresponding thermodynamic phase space is a 5 -dimensional one coordinated by the set of independent coordinates $\left\{J_{\Phi}, S, \Phi, T,-Q\right\}$. The fundamental Gibbs 1- form defined on $\mathcal{T}$ can then be written as

$$
\Theta=d J_{\Phi}-T d S+Q d \Phi .
$$

The non-degenerate metric $G$ from Eq.(3.3) can be written as

$$
G=\left(d J_{\Phi}-T d S+Q d \Phi\right)^{2}+(T S-Q \Phi)(-d S d T-d \Phi d Q) .
$$




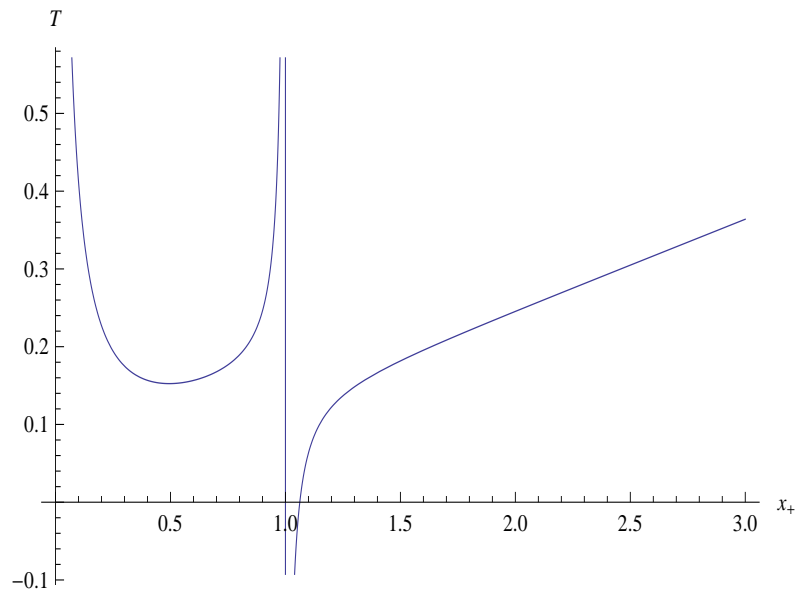

(a)

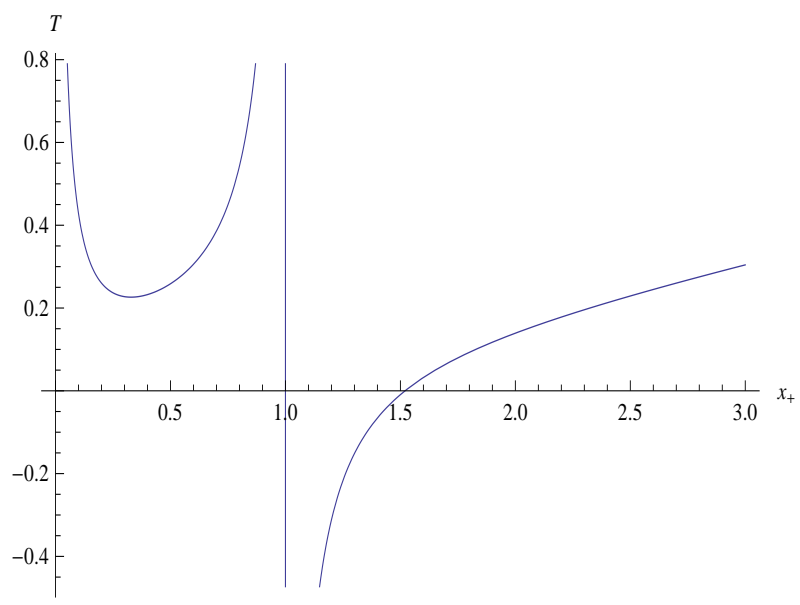

(c)

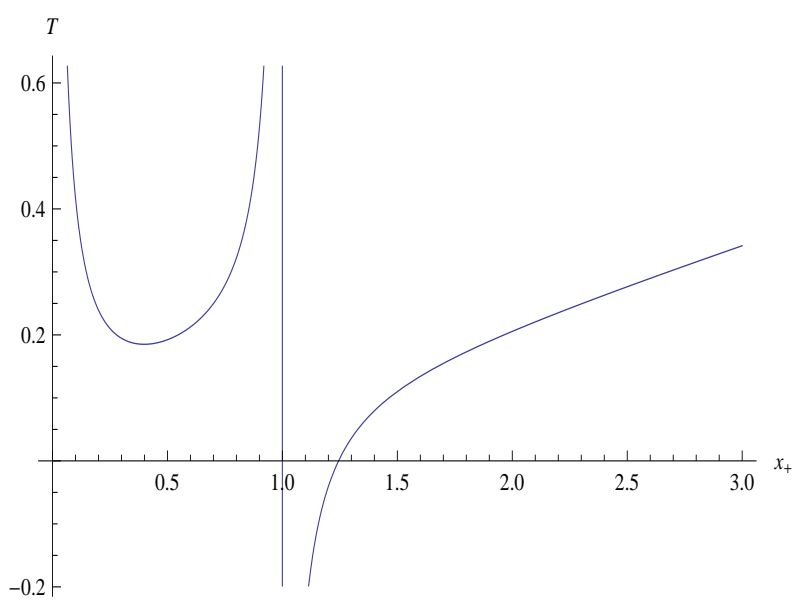

(b)

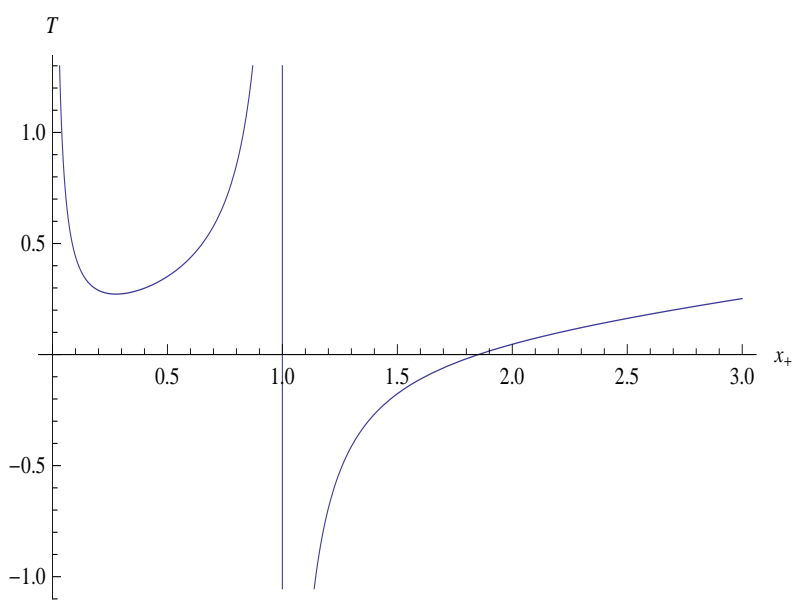

(d)

Figure 8. $T$ vs. $x_{+}$for (a) $k=-1, \Phi=1$ (b) $k=-1, \Phi=2$ (c) $k=-1, \Phi=3$ (d) $k=-1, \Phi=4$

Introducing the map

$$
\varphi:\{S, \Phi\} \mapsto\left\{J_{\Phi}(S, \Phi), S, \Phi, \frac{\partial J_{\Phi}}{\partial S}, \frac{\partial J_{\Phi}}{\partial \Phi}\right\},
$$

the space of thermodynamic equilibrium states can be induced. According to Eq.(3.4), the thermodynamic metric $g$ can be written as follows

$$
g=\left(S \frac{\partial J_{\Phi}}{\partial S}+\Phi \frac{\partial J_{\Phi}}{\partial \Phi}\right)\left(-\frac{\partial^{2} J_{\Phi}}{\partial S^{2}} d S^{2}+\frac{\partial^{2} J_{\Phi}}{\partial \Phi^{2}} d \Phi^{2}\right) .
$$




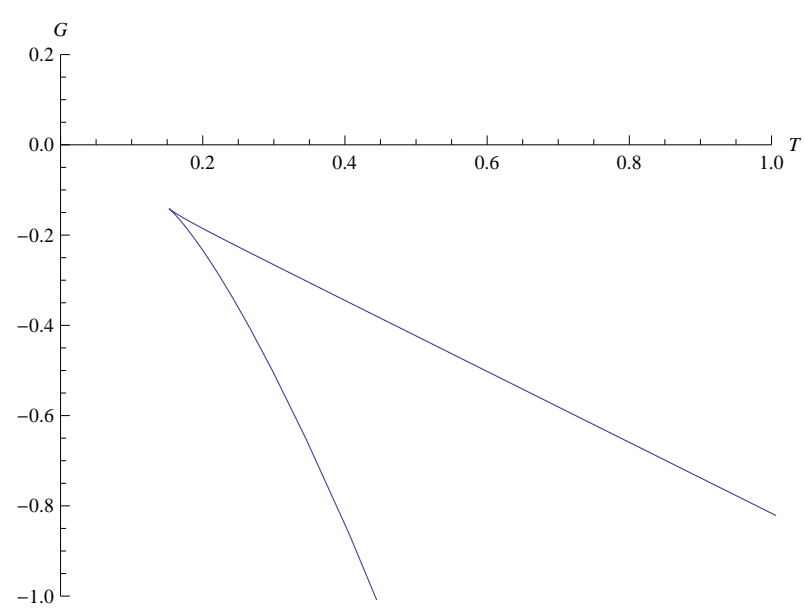

(a)

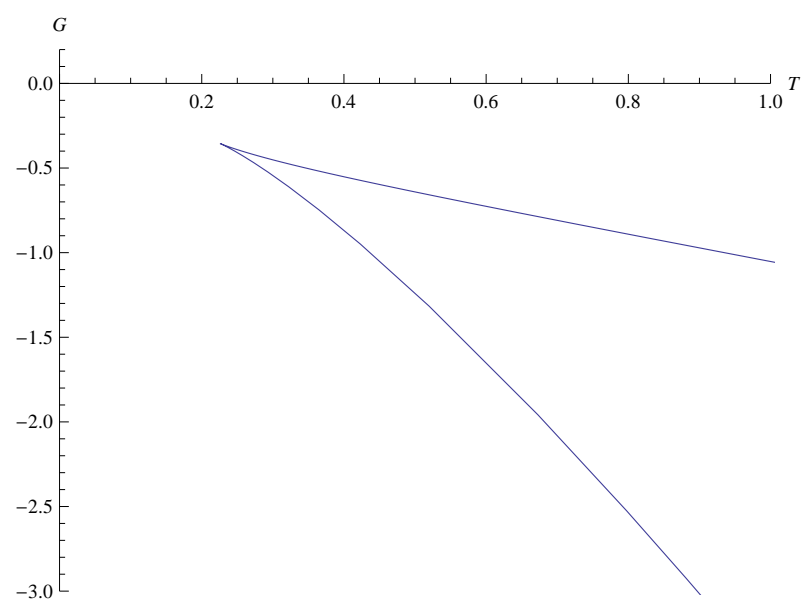

(c)

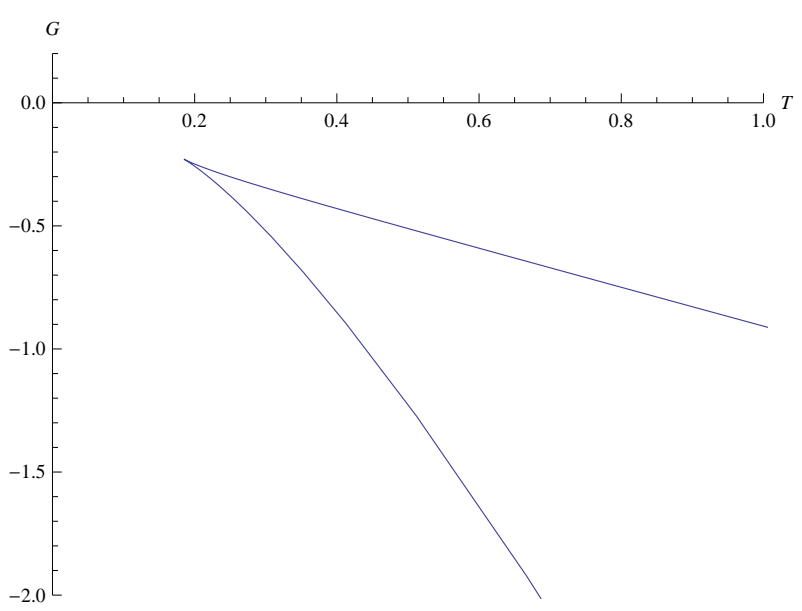

(b)

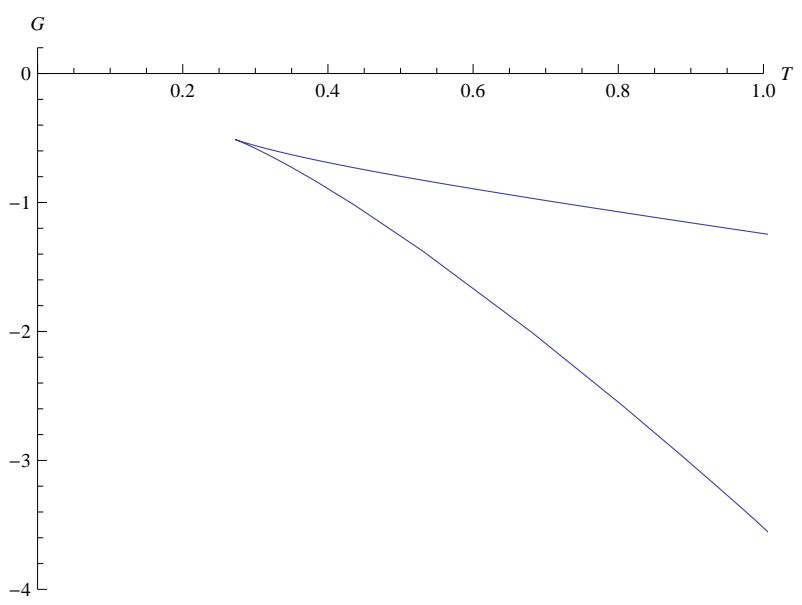

(d)

Figure 9. $G$ vs. $T$ for (a) $k=-1, \Phi=1$ (b) $k=-1, \Phi=2$ (c) $k=-1, \Phi=3$ (d) $k=-1, \Phi=4$

Utilizing Eqs.(2.4),(2.5) and (4.5), we can easily calculate the relevant quantities in Eq.(4.10)as

$$
\begin{aligned}
\frac{\partial J_{\Phi}}{\partial S} & =\frac{-\Phi^{2} x_{+}^{2}-2\left(k-3 x_{+}^{2}\right)\left(k+x_{+}^{2}\right)}{16 l^{2} \pi x_{+}\left(k+x_{+}^{2}\right)}, \\
\frac{\partial J_{\Phi}}{\partial \Phi} & =-\frac{\kappa^{2} \mu^{2} \Omega_{k} x_{+} \Phi}{16 l^{2}} \\
\frac{\partial^{2} J_{\Phi}}{\partial \Phi^{2}} & =-\frac{\kappa^{2} \mu^{2} \Omega_{k} x_{+}}{16 l^{2}}, \\
\frac{\partial^{2} J_{\Phi}}{\partial S^{2}} & =\frac{2 k^{3}+10 k^{2} x_{+}^{2}+14 k x_{+}^{4}+6 x_{+}^{6}-x_{+}^{2}\left(k-x_{+}^{2}\right) \Phi^{2}}{8 l^{2} \pi^{2} \kappa^{2} \mu^{2} \Omega_{k} x_{+}\left(k+x_{+}^{2}\right)^{3}} .
\end{aligned}
$$

Comparing Eqs.(4.11), (4.12)with Eqs.(2.3), (2.6), we can find

$$
\frac{\partial J_{\Phi}}{\partial S}=T, \quad \frac{\partial J_{\Phi}}{\partial \Phi}=-Q
$$


The results of Eq.(4.15) coincide with Eq.(4.6)exactly. Substituting Eqs.(4.11)-(4.14) into Eq.(4.10), we can find the component of the thermodynamic metric $g$ as

$$
\begin{aligned}
& g_{\Phi \Phi}=\frac{E\left(x_{+}, \Phi\right)}{1024 \pi \kappa^{2} \mu^{2} \Omega_{k} l^{4}\left(k+x_{+}^{2}\right)^{2}}, \\
& g_{S S}=-\frac{F\left(x_{+}, \Phi\right)}{512 \pi^{3} l^{4} \kappa^{2} \mu^{2} \Omega_{k} x_{+}^{2}\left(k+x_{+}^{2}\right)^{4}},
\end{aligned}
$$

where

$$
\begin{aligned}
E\left(x_{+}, \Phi\right) & =4 \pi \kappa^{4} \mu^{4} \Omega_{k}^{2} x_{+}^{2} \Phi^{2}\left(k+x_{+}^{2}\right) \\
& -\kappa^{2} \mu^{2} \Omega_{k}\left[4 k x_{+}^{2}-2 k^{2}+x_{+}^{2}\left(6 x_{+}^{2}-\Phi^{2}\right)\right]\left[S_{0}+\pi \kappa^{2} \mu^{2} \Omega_{k}\left(x_{+}^{2}+2 k \ln x_{+}\right)\right], \\
F\left(x_{+}, \Phi\right) & =\left[-2 k^{2}+4 k x_{+}^{2}+x_{+}^{2}\left(6 x_{+}^{2}-\Phi^{2}\right)\right] \times\left[4 S_{0}+\pi \kappa^{2} \mu^{2} \Omega_{k}\left(x_{+}^{2}+2 k \ln x_{+}\right)\right] \\
& -4 \pi \kappa^{2} \mu^{2} \Omega_{k} \Phi^{2} x_{+}^{2}\left(k+x_{+}^{2}\right)\left[2 k^{3}+10 k^{2} x_{+}^{2}+k x_{+}^{2}\left(14 x_{+}^{2}-\Phi^{2}\right)+x_{+}^{4}\left(6 x_{+}^{2}+\Phi^{2}\right)\right] .
\end{aligned}
$$

So the Legendre invariant scalar curvature can be given as

$$
\mathfrak{R}_{\Phi}=\frac{W\left(x_{+}, \Phi\right)}{Y\left(x_{+}, \Phi\right)}
$$

where

$$
\begin{aligned}
Y\left(x_{+}, \Phi\right)= & \kappa^{2} \mu^{2} \Omega_{k}\left[2 k^{3}+10 k^{2} x_{+}^{2}+k x_{+}^{2}\left(14 x_{+}^{2}-\Phi^{2}\right)+x_{+}^{4}\left(6 x_{+}^{2}+\Phi^{2}\right)\right]^{2} \\
& \times\left\{4 S_{0}\left[2 k^{2}-4 k x_{+}^{2}+x_{+}^{2}\left(\Phi^{2}-6 x_{+}^{2}\right)\right]+2 k \pi \kappa^{2} \mu^{2} \Omega_{k}\left[2 k^{2}-4 k x_{+}^{2}+x_{+}^{2}\left(\Phi^{2}-6 x_{+}^{2}\right)\right] \ln x_{+}\right. \\
& \left.\left.+\pi^{2} \kappa^{2} \mu^{2} \Omega_{k} x_{+}^{2}\left[2 k^{2}-x_{+}^{2}\left(6 x_{+}^{2}-5 \Phi^{2}\right)+4 k \Phi^{2}\right)\right]\right\}^{3} .
\end{aligned}
$$

From Eq.(4.21), we can find that the Legendre invariant scalar curvature may diverge when $2 k^{3}+10 k^{2} x_{+}^{2}+k x_{+}^{2}\left(14 x_{+}^{2}-\Phi^{2}\right)+x_{+}^{4}\left(6 x_{+}^{2}+\Phi^{2}\right)=0$, which coincides with Eq.(4.2). That is the exact point where the phase transition may take place. To get an intuitive sense on this issue, we plot Figure 10, which shows the correpondence of the divergence of specific heat $C_{\Phi}$ and the thermodynamic scalar curvature of $\mathfrak{R}_{\Phi}$. From Figure 10, we learn that the Legendre invariant metric constructed in geometrothermodynamics correctly produces the behavior of the thermodynamic interaction and phase transition structure.

\section{Analytical check of Ehrenfest equations in the fixed-potential ensemble}

To investigate the grand-canonical ensemble phase transition, we would like to carry out an analytical check of Ehrenfest equations for this case. In conventional thermodynamics, Ehrenfests equations are given as

$$
\begin{aligned}
& \left(\frac{\partial P}{\partial T}\right)_{S}=\frac{C_{P_{2}}-C_{P_{1}}}{V T\left(\alpha_{2}-\alpha_{1}\right)}=\frac{\Delta C_{P}}{V T \Delta \alpha} \\
& \left(\frac{\partial P}{\partial T}\right)_{V}=\frac{\alpha_{2}-\alpha_{1}}{\kappa_{T_{2}}-\kappa_{T_{1}}}=\frac{\Delta \alpha}{\Delta \kappa_{T}} .
\end{aligned}
$$




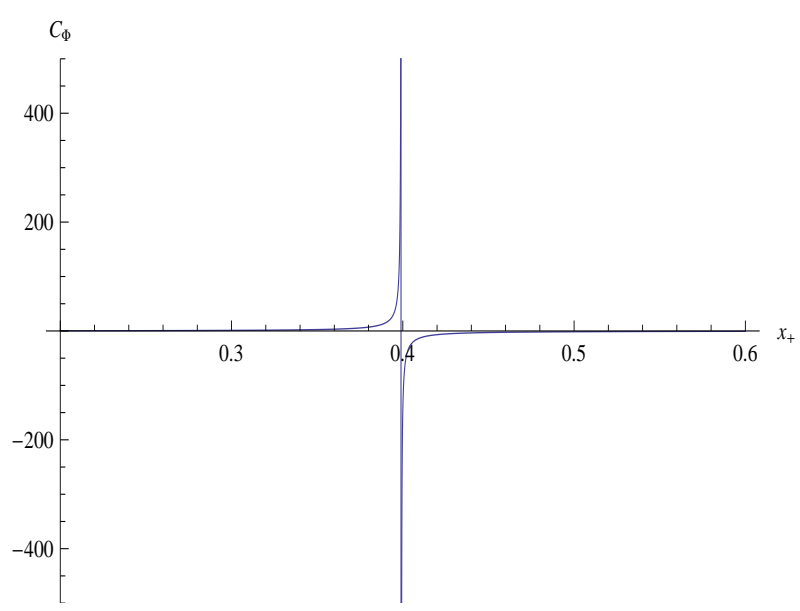

(a)

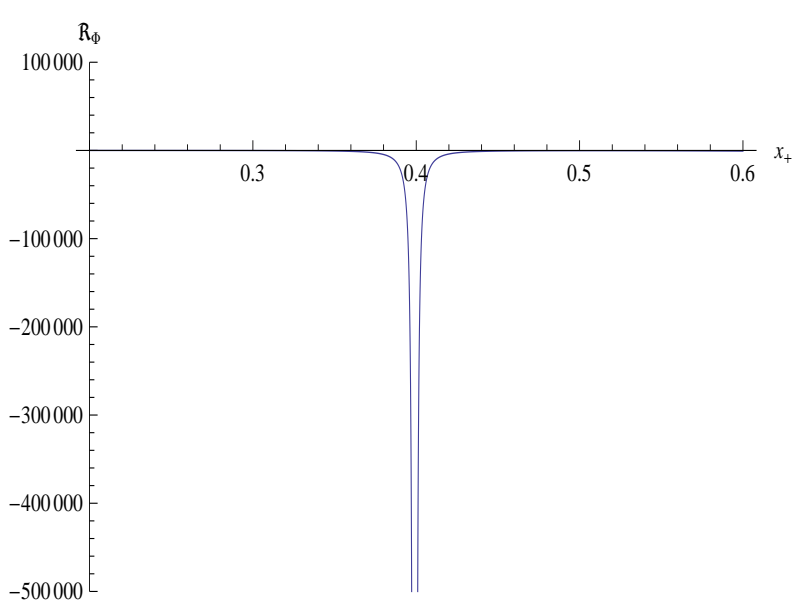

(b)

Figure 10. (a) $C_{\Phi}$ vs. $x_{+}$for $k=-1, \Phi=2(b) \mathfrak{R}_{\Phi}$ vs. $x_{+}$for $k=-1, \Phi=2$

Taking the similar approach as Ref.[28] and considering the analogy $(V \leftrightarrow Q, P \leftrightarrow-\Phi)$ between the thermodynamic state variables and black hole parameters, we can easily write down the corresponding Ehrenfests equations for the charged topological black hole as

$$
\begin{aligned}
& -\left(\frac{\partial \Phi}{\partial T}\right)_{S}=\frac{C_{\Phi_{2}}-C_{\Phi_{1}}}{T Q\left(\alpha_{2}-\alpha_{1}\right)}=\frac{\Delta C_{\Phi}}{T Q \Delta \alpha}, \\
& -\left(\frac{\partial \Phi}{\partial T}\right)_{Q}=\frac{\alpha_{2}-\alpha_{1}}{\kappa_{T_{2}}-\kappa_{T_{1}}}=\frac{\Delta \alpha}{\Delta \kappa_{T}},
\end{aligned}
$$

where $\alpha=\frac{1}{Q}\left(\frac{\partial Q}{\partial T}\right)_{\Phi}$ is the analog of volume expansion coefficient and $\kappa_{T}=\frac{1}{Q}\left(\frac{\partial Q}{\partial \Phi}\right)_{T}$ is the analog of isothermal compressibility. Utilizing Eqs.(2.3)-(2.6), we can derive the explicit forms of relevant quantities as

$$
\begin{aligned}
\alpha & =\frac{1}{Q}\left(\frac{\partial Q}{\partial T}\right)_{\Phi}=\frac{16 l^{2} \pi\left(k+x_{+}^{2}\right)^{2} x_{+}}{2 k^{3}-k \Phi^{2} x_{+}^{2}+10 k^{2} x_{+}^{2}+\Phi^{2} x_{+}^{4}+14 k x_{+}^{4}+6 x_{+}^{6}}, \\
\kappa_{T} & =\frac{1}{Q}\left(\frac{\partial Q}{\partial \Phi}\right)_{T}=\frac{\left(\Phi^{2} x_{+}^{2}+2\left(k+x_{+}^{2}\right)^{2}\right)\left(k+3 x_{+}^{2}\right)}{\Phi\left(2 k^{3}-k \Phi^{2} x_{+}^{2}+10 k^{2} x_{+}^{2}+\Phi^{2} x_{+}^{4}+14 k x_{+}^{4}+6 x_{+}^{6}\right)} .
\end{aligned}
$$

It is quite interesting to note that $C_{\Phi}, \alpha, \kappa_{T}$ share the same factor in their denominators, namely $\left(2 k^{3}-k \Phi^{2} x_{+}^{2}+10 k^{2} x_{+}^{2}+\Phi^{2} x_{+}^{4}+14 k x_{+}^{4}+6 x_{+}^{6}\right)$, which implies that $\alpha, \kappa_{T}$ may also diverge at the critical point. To witness the divergence of $\alpha, \kappa_{T}$, we plot them in Figure 11.

Now let's embark on checking the validity of Ehrenfests equations (5.3)-(5.4) at the critical point. Note that

$$
Q \alpha=\left(\frac{\partial Q}{\partial T}\right)_{\Phi}=\left(\frac{\partial Q}{\partial S}\right)_{\Phi}\left(\frac{\partial S}{\partial T}\right)_{\Phi}=\left(\frac{\partial Q}{\partial S}\right)_{\Phi}\left(\frac{C_{\Phi}}{T}\right),
$$

then the R.H.S of Eq.(5.3) can be transformed into

$$
\frac{\Delta C_{\Phi}}{T Q \Delta \alpha}=\left[\left(\frac{\partial S}{\partial Q}\right)_{\Phi}\right]_{c r i}
$$




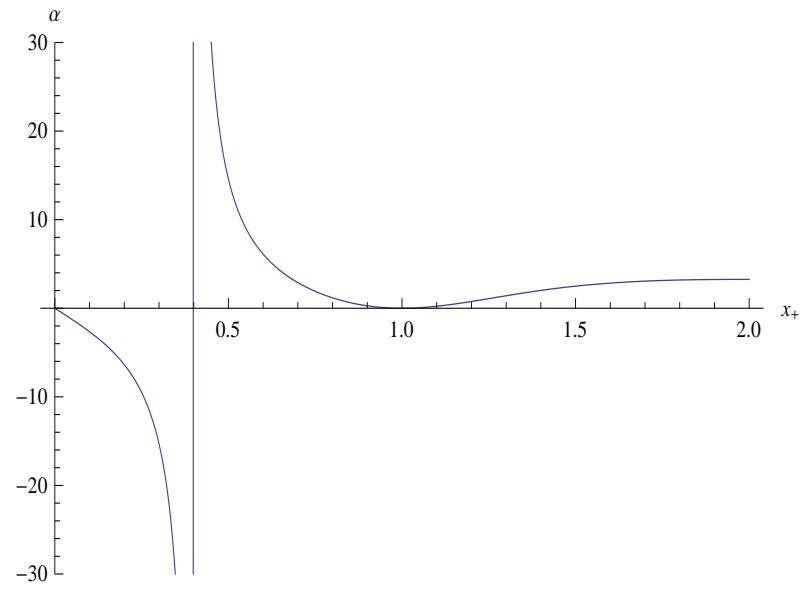

(a)

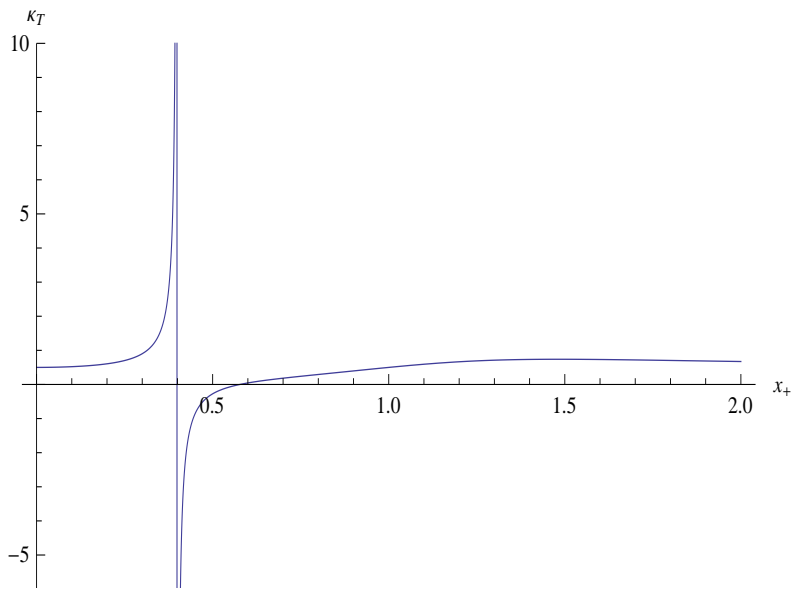

(b)

Figure 11. (a) $\alpha$ vs. $x_{+}$for $k=-1, \Phi=2(b) \kappa_{T}$ vs. $x_{+}$for $k=-1, \Phi=2$

where the subscript "cri" denotes the value at the critical point. Utilizing Eqs.(2.4)-(2.6) and (5.8), we obtain

$$
\frac{\Delta C_{\Phi}}{T Q \Delta \alpha}=\frac{8 \pi l^{2}\left(k+x_{+c}^{2}\right)}{\Phi x_{+c}}
$$

where $x_{+c}$ denotes the value of $x_{+}$at the critical point. The L.H.S of Eq.(5.3) can be derived as

$$
-\left[\left(\frac{\partial \Phi}{\partial T}\right)_{S}\right]_{c r i}=\frac{8 \pi l^{2}\left(k+x_{+c}^{2}\right)}{\Phi x_{+c}} .
$$

From Eqs.(5.9) and (5.10), we can draw the conclusion that the first equation of Erhenfest equations is valid at the critical point. The L.H.S of Eq.(5.4) can be obtained as

$$
-\left[\left(\frac{\partial \Phi}{\partial T}\right)_{Q}\right]_{c r i}=\frac{16 \pi l^{2} \Phi x_{+c}\left(k+x_{+c}^{2}\right)^{2}}{\left(k+3 x_{+c}^{2}\right)\left(\Phi^{2} x_{+c}^{2}+2\left(k+x_{+c}^{2}\right)^{2}\right.} .
$$

From the thermodynamic identity [28]

$$
\left(\frac{\partial Q}{\partial \Phi}\right)_{T}\left(\frac{\partial \Phi}{\partial T}\right)_{Q}\left(\frac{\partial T}{\partial Q}\right)_{\Phi}=-1
$$

we can derive that

$$
Q \kappa_{T}=\left(\frac{\partial Q}{\partial \Phi}\right)_{T}=-\left(\frac{\partial T}{\partial \Phi}\right)_{Q}\left(\frac{\partial Q}{\partial T}\right)_{\Phi}=-\left(\frac{\partial T}{\partial \Phi}\right)_{Q} Q \alpha
$$

from which we can calculate the R.H.S of Eq.(5.4) and get

$$
\frac{\Delta \alpha}{\Delta \kappa_{T}}=-\left[\left(\frac{\partial \Phi}{\partial T}\right)_{Q}\right]_{c r i}=\frac{16 \pi l^{2} \Phi x_{+c}\left(k+x_{+c}^{2}\right)^{2}}{\left(k+3 x_{+c}^{2}\right)\left(\Phi^{2} x_{+c}^{2}+2\left(k+x_{+c}^{2}\right)^{2}\right]} .
$$

Eqs.(5.11) and (5.14) reveal the validity of the second equation of Ehrenfest equations. So far, we have proved that both the Ehrenfest equations are correct at the critical point. 
Utilizing Eq.(4.2), we can prove that Eqs.(5.9)and (5.14) has the same value at the critical point. And the Prigogine-Defay $(\mathrm{PD})$ ratio is

$$
\Pi=\frac{\Delta C_{\Phi} \Delta \kappa_{T}}{T_{c} Q(\Delta \alpha)^{2}}=1 .
$$

Eq.(5.15) and the validity of Ehrenfest equations show that the grand-canonical ensemble phase transition of the charged topological black hole in HL gravity is a second order transition. It is necessary to emphasize that although $C_{\Phi}, \alpha, \kappa_{T}$ diverge at the critical point, they can cancel each other and allow the R.H.S of Eqs.(5.3) and (5.4) to be finite.

\section{Conclusions}

The phase transition of a charged topological black hole in Hořava-Lifshitz gravity has been investigated in fixed-charge ensemble and fixed-potential ensemble respectively. To build up geometrothermodynamics in both ensembles, we choose the corresponding thermodynamic potential and build up both thermodynamic phase space and the space of thermodynamic equilibrium states. In the fixed-charge ensemble, the critical point at which the specific heat $C_{Q}$ diverges wittnesses the divergence of the corresponding Legendre invariant thermodynamic scalar curvature $\mathfrak{R}_{Q}$. In the fixed-potential ensemble, the critical point at which specific heat $C_{\Phi}$ diverges also wittnesses the divergence of the corresponding Legendre invariant thermodynamic scalar curvature $\mathfrak{R}_{\Phi}$. Based on the above results, we can surely conclude that the Legendre invariant metrics constructed in geometrothermodynamics can correctly produce the behavior of the thermodynamic interaction and phase transition structure, no matter which ensemble is chosen. The Legendre invariant metrics constructed here have successfully predicted the phase transition of the charged topological black hole in H-L gravity while other metrics failed [21], where Cao et al. regarded it as "one exception".

The concrete phase transition structures are different due to the choice of ensemble, but both of them can build up geometrothermodynamics. The research in both complementary ensembles allows us to investigate the phase transition from different perspectives and gain a unified picture. We have discovered for the first time the phase transition of a charged topological black hole in Hořava-Lifshitz gravity in the grand-canonical ensemble. It is similar to the canonical ensemble where the phase transition only takes place for $k=-1$. However it is found that the location of the phase transition point depends on the value of potential, which is different from the canonical ensemble where the phase transition point is independent of the parameters. After an analytical check of Ehrenfest scheme, we find that the new phase transition is a second order one.

It is worth noting that the dependence of the thermodynamic scalar curvature on the ensemble does not contradict the property of Legendre invariance. Firstly, the phase structures are different due to different behaviors of the specific heat. This phenomenon should be attributed to different boundary conditions corresponding to different choice of ensemble. The stability of black holes turns out to depend on the choice of boundary conditions and consequently on the ensemble [70]. Secondly, the motivation of thermodynamic geometry 
method is to find the relation between the divergence of thermodynamic curvature scalar and the existence of phase transition. So a good thermodynamic metric should reproduce the behavior of the system no matter which ensemble is chosen. Since the phase structures are different in different ensembles, we should not expect the thermodynamic scalar curvature keep invariant. Recently, the founder of geometrothermodynamics Quevedo [70] also discussed the ensemble dependence of geometrothermodynamics. In that paper, the concepts of "total Legendre transformations" and "partial Legendre transformations" were put forward. If all the extensive variables change through Legendre transformation, this kind of Legendre transformation has been called "total Legendre transformation". Otherwise, if some extensive variables change through Legendre transformation while others keep the same, this kind of Legendre transformation has been called "partial Legendre transformation". The word "partial" means only some (not all) extensive variables undergo Legendre transformation. The transformation that relates the potentials $M(S, Q)$ and $H(S, \phi)$ can serve as an example of "partial Legendre transformation" because only the extensive variable $Q$ is transformed into $\phi$ while the the extensive variable $S$ remains the same during the transformation. The transformation that relates the potentials $M(S, Q)$ and $G(T, \phi)$ can serve as an example of "total Legendre transformation" because both of two extensive variables $S$ and $Q$ are transformed into $T$ and $\phi$ respectively during the transformation. Two types of metrics were investigated in that paper. It has been proved that a metric which is invariant under partial Legendre transformation can not be used to distinguish the thermodynamic properties of different ensembles naturally. On the contrary, the metric that is only invariant under a total Legendre transformation can reasonably be used to distinguish the thermodynamic properties of different ensembles. In our paper, we have used the metric only invariant under total Legendre transformation and it has described the black hole thermodynamics and phase transition successfully.

In the end, we would like to talk more about the interesting phase transition phenomenon in Hořava-Lifshitz gravity. The phase structures in the charged topological black hole are quite different from that in Einstein gravity. The phase transition takes place only for $k=-1$ in both complementary ensembles while in Einstein theory, only $k=1$ case exhibits such phase transition. This may be attributed to the ultraviolet behaviour of spacetime in Horava-Lifshitz gravity, as argued by Cao et al. [21].

\section{Acknowledgments}

We would like to give great thanks to the anonymous referee for his helpful suggestions about this paper. This research is supported by the National Natural Science Foundation of China (Grant Nos.11235003, 11175019, 11178007). It is also supported by "Thousand

Hundred Ten" project of Guangdong Province and Natural Science Foundation of Zhanjiang Normal University (Grant No. QL1104).

\section{References}

[1] J. D. Bekenstein, Black Holes and Entropy, Phys. Rev. D 7 (1973) 2333. 
[2] S.W. Hawking, Particle creation by black holes,Commum. Math. Phys. 43 (1975) 199.

[3] S.W. Hawking and D.N. Page, Thermodynamics of black holes in anti-de Sitter space, Comm. Math. Phys. 87 (1983) 577.

[4] A. Chamblin, R. Emparan, C.V. Johnson and R.C. Myers, Charged AdS Black Holes and Catastrophic Holography, Phys. Rev. D 60 (1999) 064018 [arXiv:hep-th/9902170].

[5] A. Chamblin, R. Emparan, C.V. Johnson and R.C. Myers, Holography, Thermodynamics and Fluctuations of Charged AdS Black Holes, Phys. Rev. D 60 (1999) 104026 [arXiv:hep-th/9904197].

[6] M. M. Caldarelli, G. Cognola and D. Klemm, Thermodynamics of Kerr-Newman-AdS Black Holes and Conformal Field Theories, Class. Quantum Grav. 17 (2000) 399-420 [arXiv:hep-th/9908022].

[7] S. Nojiri and S. D. Odintsov, Anti-de Sitter Black Hole Thermodynamics in Higher Derivative Gravity and New Confining-Deconfining Phases in dual CFT, Phys. Lett. B 521, (2001) 87-95 [arXiv:hep-th/0109122].

[8] R. G. Cai, Gauss-Bonnet Black Holes in AdS Spaces, Phys. Rev. D 65 (2002) 084014 [arXiv:hep-th/0109133].

[9] M. Cveti č, S. Nojiri and S. D. Odintsov, Black Hole Thermodynamics and Negative Entropy in deSitter and Anti-deSitter Einstein-Gauss-Bonnet gravity, Nucl. Phys. B 628 (2002) 295-330 [arXiv:hep-th/0112045].

[10] S. Carlip and S. Vaidya, Phase Transitions and Critical Behavior for Charged Black Holes, Class. Quantum Grav. 20 (2003) 3827-3838 [arXiv:gr-qc/0306054].

[11] R. G. Cai and A. Wang, Thermodynamics and Stability of Hyperbolic Charged Black Holes, Phys. Rev. D 70 (2004) 064013 [arXiv:hep-th/0406057].

[12] Y. S. Myung, No Hawking-Page phase transition in three dimensions, Phys. Lett. B 624 (2005) 297 [arXiv:hep-th/0506096].

[13] B. M. N. Carter and I. P. Neupane, Thermodynamics and Stability of Higher Dimensional Rotating (Kerr) AdS Black Holes, Phys. Rev. D 72 (2005) 043534 [arXiv:gr-qc/0506103].

[14] R. G. Cai, S.P. Kim and B. Wang, Ricci Flat Black Holes and Hawking-Page Phase Transition in Gauss-Bonnet Gravity and Dilaton Gravity, Phys. Rev. D 76 (2007) 024011 [arXiv:0705.2469v1].

[15] Y. S. Myung, Y.W. Kim and Y.J. Park, Thermodynamics and phase transitions in the Born-Infeld-anti-de Sitter black holes, Phys. Rev. D 78 (2008) 084002 [arXiv:0805.0187v2].

[16] Y. S. Myung, Phase transition between non-extremal and extremal Reissner-Nordstrom black holes, Mod.Phys.Lett. A 23 (2008) 667-676 [arXiv:0710.2568v3].

[17] G. Koutsoumbas, E. Papantonopoulos and G. Siopsis, Phase Transitions in Charged Topological-AdS Black Hole, JHEP 0805 (2008) 107 [arXiv:0801.4921v2].

[18] M. Cadoni, G. D. Appollonio and P. Pani, Phase transitions between Reissner-Nordstrom and dilatonic black holes in 4D AdS spacetime, JHEP 1003 (2010) 100 [arXiv:0912.3520v3].

[19] H. S. Liu, H. Lu, M. Luo and K. N. Shao, Thermodynamical Metrics and Black Hole Phase Transitions, JHEP 1012 (2010) 054 [arXiv:1008.4482].

[20] A. Sahay, T. Sarkar and G. Sengupta, On The Phase Structure and Thermodynamic Geometry of R-Charged Black Holes, JHEP 1011 (2010) 125 [arXiv:1009.2236]. 
[21] Q. J. Cao, Y. X. Chen and K. N. Shao, Black hole phase transitions in Horaava-Lifshitz gravity, Phys. Rev. D 83 (2011) 064015 [arXiv:1010.5044v2].

[22] H. Quevedo, A. Sanchez and S. Taj, Thermodynamics of topological black holes in Hořava-Lifshitz gravity, J. Phys.Phy:Conf.Ser 354 (2012) 012015.

[23] H. Quevedo, A. Sanchez, S. Taj and A. Vazquez, Geometrothermodynamics in Hor̆ava-Lifshitz gravity, J. Phys.A-Math.Theor 45 (2012) 055211.

[24] R. Banerjee, S. K. Modak and S. Samanta, Glassy Phase Transition and Stability in Black Holes, Eur. Phys. J. C 70 (2010) 317 [arXiv:1002.0466].

[25] R. Banerjee, S. K. Modak and S. Samanta, Second Order Phase Transition and Thermodynamic Geometry in Kerr-AdS Black Hole, Phys. Rev. D 84 (2011) 064024 [arXiv:1005.4832].

[26] R. Banerjee and D. Roychowdhury, Critical phenomena in Born-Infeld AdS black holes, Phys. Rev. D 85 (2011) 044040 [arXiv:1111.0147].

[27] R. Banerjee, S. Ghosh and D. Roychowdhury, New type of phase transition in Reissner Nordstrom - AdS black hole and its thermodynamic geometry, Phys. Lett. B 696, 156 (2011) 156 [arXiv:1008.2644].

[28] R. Banerjee and D. Roychowdhury, Thermodynamics of phase transition in higher dimensional AdS black holes, JHEP 11 (2011) 004 [arXiv:1109.2433].

[29] R. Banerjee, S. K. Modak and D. Roychowdhury, A unified picture of phase transition: from liquid-vapour systems to AdS black holes, JHEP 1210 (2012) 125 [arXiv:1106.3877].

[30] S. W. Wei and Y. X. Liu, Thermodynamic Geometry of black hole in the deformed Horava-Lifshitz gravity, Europhys.Lett. 99 (2012) 20004 [arXiv:1002.1550].

[31] B. R. Majhi and D. Roychowdhury, Phase transition and scaling behavior of topological charged black holes in Horava-Lifshitz gravity, Class. Quantum Grav. 29 (2012) 245012 [arXiv:1205.0146].

[32] W. Kim and Y. Kim, Phase transition of quantum corrected Schwarzschild black hole, Phys. Lett. B 718 (2012) 687-691 [arXiv:1207.5318].

[33] Y. D. Tsai, X. N. Wu and Y. Yang, Phase Structure of Kerr-AdS Black Hole, Phys. Rev. D 85 (2012) 044005 [arXiv:1104.0502].

[34] F. Capela and G. Nardini, Hairy Black Holes in Massive Gravity: Thermodynamics and Phase Structure, Phys. Rev. D 86 (2012) 024030 [arXiv:1203.4222].

[35] D. Kubiznak and R. B. Mann, P-V criticality of charged AdS black holes, JHEP 1207 (2012)033 [arXiv:1205.0559].

[36] S. W. Wei and Y. X. Liu, Critical phenomena and thermodynamic geometry of charged Gauss-Bonnet AdS black holes, Phys. Rev. D 87 (2013) 044014 [arXiv:1209.1707].

[37] M. Eune, W. Kim and S. H. Yi, Hawking-Page phase transition in BTZ black hole revisited, JHEP 1303 (2013) 020 [arXiv:1301.0395].

[38] F. Weinhold, Metric geometry of equilibrium thermodynamics, Chem.Phys. 63 (1975) 2479.

[39] G. Ruppeiner, A Riemannian geometric model, Phys. Rev. A 20 (1979) 1608.

[40] P. Salamon, E. Ihrig and R. S. Berry, A group of coordinate transformations which preserve the metric of Weinhold, J. Math. Phys. 24 (1983)2515. 
[41] R. Mrugala, J. D. Nulton, J. C. Schon, and P. Salamon, Statistical approach to the geometric structure of thermodynamics, Phys. Rev. A 41 (1990) 3156.

[42] H. Quevedo, Geometrothermodynamics, J. Math. Phys. 48 (2007) 013506 [arXiv:physics/0604164].

[43] H. Quevedo, Geometrothermodynamics of black holes, Gen.Rel.Grav. 40 (2008) 971-984 [arXiv:0704.3102].

[44] H. Quevedo and A. Sanchez, Geometrothermodynamics of asymptotically anti - de Sitter black holes, JHEP 0809 (2008) 034 [arXiv:0805.3003].

[45] J. L. Alvarez, H. Quevedo and A. Sanchez, Unified geometric description of black hole thermodynamics, Phys. Rev. D 77 (2008) 084004 [arXiv:0801.2279].

[46] H. Quevedo and A. Sanchez, Geometrothermodynamics of black holes in two dimensions, Phys. Rev. D 79 (2009) 087504 [arXiv:0902.4488].

[47] H. Quevedo and A. Sanchez, Geometric description of BTZ black holes thermodynamics, Phys. Rev. D 79 (2009) 024012 [arXiv:0811.2524].

[48] M. Akbar, H. Quevedo, K. Saifullah and A. Sanchez, S. Taj, Thermodynamic Geometry Of Charged Rotating BTZ Black Holes, Phys. Rev. D 83 (2011) 084031 [arXiv:1101.2722].

[49] H. Quevedo, A. Sanchez, S. Taj and A. Vazquez, Phase transitions in geometrothermodynamics, Gen.Rel. Grav 43 (2011) 1153-1165 [arXiv:1010.5599].

[50] A. Aviles, A. B. Almodovar, L.Campuzano and H. Quevedo, Extending the generalized Chaplygin gas model by using geometrothermodynamics, Phys. Rev. D 86 (2012) 063508 [arXiv:1203.4637].

[51] Y. W. Han and G. Chen, Thermodynamics, geometrothermodynamics and critical behavior of (2+1)-dimensional black hole, Phys. Lett. B 714 (2012) 127-130 [arXiv:1207.5626].

[52] P. Horřava, Quantum Gravity at a Lifshitz Point, Phys. Rev. D 79 (2009) 084008 [arXiv:0901.3775].

[53] P. Horřava, Membranes at Quantum Criticality, JHEP 0903 (2009) 020 [arXiv:0812.4287].

[54] P. Horřava, Spectral Dimension of the Universe in Quantum Gravity at a Lifshitz Point, Phys. Rev. Lett 102 (2009) 161301 [arXiv:0902.3657].

[55] H. Lu, J. Mei and C. N. Pope, Solutions to Horava Gravity, Phys. Rev. Lett 103 (2009) 091301 [arXiv:0904.1595].

[56] R. G. Cai, L. M. Cao and N. Ohta, Topological Black Holes in Horava-Lifshitz Gravity, Phys. Rev. D 80 (2009) 024003 [arXiv:0904.3670].

[57] E. O. Colgain and H.Yavartanoo, Dyonic solution of Horava-Lifshitz Gravity, JHEP 0908 (2009) 021 [arXiv:0904.4357].

[58] A.Kehagias and K.Sfetsos, The black hole and FRW geometries of non-relativistic gravity, Phys. Lett. B 678 (2009) 123-126 [arXiv:0905.0477].

[59] E. Kiritsis, Spherically symmetric solutions in modified Horava-Lifshitz gravity, Phys. Rev. D 81 (2010) 044009 [arXiv:0911.3164].

[60] R. G. Cai, L. M. Cao and N.Ohta, Thermodynamics of Black Holes in Horava-Lifshitz Gravity, Phys. Lett. B 679 (2009) 504-509 [arXiv:0905.0751]. 
[61] R. G. Cai and N. Ohta, Horizon Thermodynamics and Gravitational Field Equations in Horava-Lifshitz Gravity, Phys. Rev. D 81 (2010) 084061 [arXiv:0910.2307].

[62] D. Y. Chen, H. T. Yang and X. T. Zu, Hawking radiation of black holes in the $z=4$ Horava-Lifshitz gravity, Phys. Lett. bf B 681 (2009) 463 [arXiv:0910.4821].

[63] X. Gao, Y. Wang, R. Brandenberger and A. Riotto, Fluctuations in a Hořava-Lifshitz Bouncing Cosmology, Phys. Rev. D 81 (2010) 083508 [arXiv:0911.3196].

[64] A. Castillo and A. Larranaga, Entropy for Black Holes in the Deformed Hořava-Lifshitz Gravity, Electron. J. Theor. Phys. 8 (2011) 1-10 [arXiv:0906.4380].

[65] B. R. Majhi, Hawking radiation and black hole spectroscopy in Horava-Lifshitz gravity, Phys. Lett. B 686 (2010) 49 [arXiv:0911.3239].

[66] J. J. Peng and S. Q. Wu, Hawking Radiation of Black Holes in Infrared Modified Hořava-Lifshitz Gravity, Eur. Phys. J. C 66 (2010) 325-331 [arXiv:0906.5121].

[67] Y. S. Myung, Entropy of black holes in the deformed Hor̆ava-Lifshitz gravity, Phys. Lett. B 684 (2010) 158 [arXiv:0908.4132].

[68] Y. S. Myung and Y. W. Kim, Thermodynamics of Hořava-Lifshitz black holes, Eur. Phys. J. C 68 (2010) 265-270 [arXiv:0905.0179].

[69] Y. S. Myung, Y. W. Kim and Y. J. Park, Ruppeiner geometry and 2D dilaton gravity in the thermodynamics of black holes, Phys.Lett. B 663 (2008) 342-350 [arXiv:0802.2152].

[70] H. Quevedo, A. Sanchez and S. Taj, On the ensemble dependence in black hole geometrothermodynamics, [arXiv:1304.3954v1]. 